AUTHOR QUERY FORM

\begin{tabular}{|l|l|l|}
\hline & Journal: JASR & Please e-mail or fax your responses and any corrections to: \\
$\begin{array}{l}\text { E. } \\
\text { ELSEVIER }\end{array}$ & Article Number: 10886 & Fax: +31204852799 \\
\hline
\end{tabular}

Dear Author,

Please check your proof carefully and mark all corrections at the appropriate place in the proof (e.g., by using on-screen annotation in the PDF file) or compile them in a separate list. Note: if you opt to annotate the file with software other than Adobe Reader then please also highlight the appropriate place in the PDF file. To ensure fast publication of your paper please return your corrections within 48 hours.

For correction or revision of any artwork, please consult http://www.elsevier.com/artworkinstructions.

Any queries or remarks that have arisen during the processing of your manuscript are listed below and highlighted by flags in the proof. Click on the ' $Q$ ' link to go to the location in the proof.

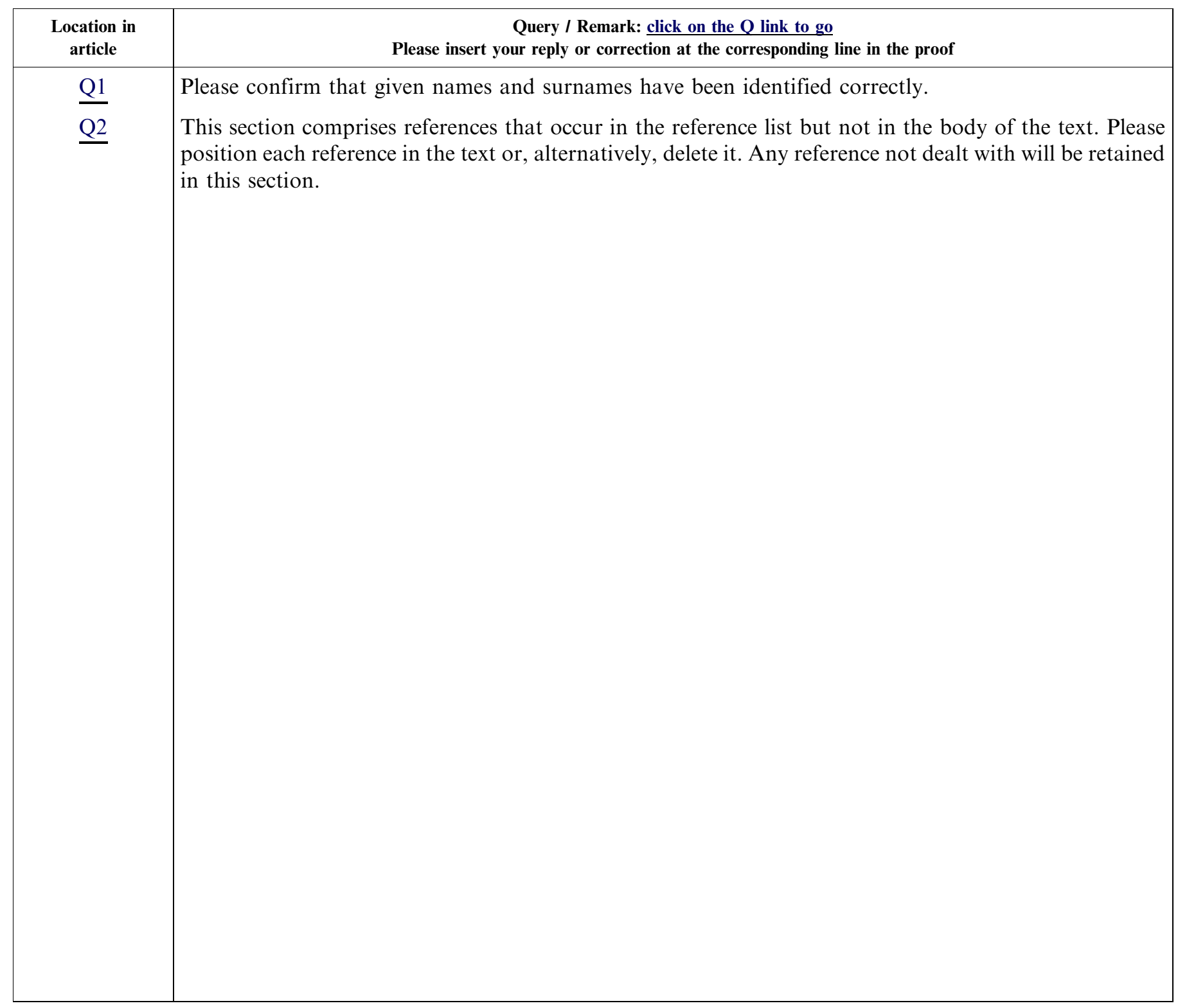

Thank you for your assistance. 


\title{
Coupled ocean-atmosphere radiative transfer model in the framework of software package SCIATRAN: Selected comparisons to model and satellite data
}

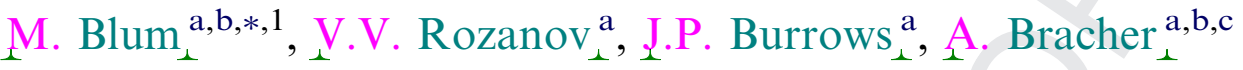 \\ ${ }^{a}$ Institute of Environmental Physics, University of Bremen, P.O. Box 330440, D-28334 Bremen, Germany \\ ${ }^{\mathrm{b}}$ Helmholtz University, Young Investigators Group PHYTOOPTICS, Germany \\ ${ }^{c}$ Alfred-Wegener-Institute for Polar and Marine Research, Bussestrasse 24, D-27570 Bremerhaven, Germany
}

Received 31 March 2011; received in revised form 9 February 2012; accepted 13 February 2012

Abstract

In order to accurately retrieve data products of importance for ocean biooptics and biogeochemistry an accurate ocean-atmosphere radiative transfer model is required. For these purposes the software package SCIATRAN, developed initially for the modeling of radiative transfer processes in the terrestrial atmosphere, was extended to account for the radiative transfer within the water and the interaction of radiative processes in the atmosphere and ocean. The extension was performed by taking radiative processes at the atmosphere-water interface, as well as within water accurately into account. Comparison results obtained with extended SCIATRAN version to predictions of other radiative transfer models and MERIS satellite spectra are presented in this paper along with a description of implemented inherent optical parameters and numerical technique used to solve coupled ocean-atmosphere radiative transfer equation. The extended version of SCIATRAN software package along with detailed User's Guide are freely distributed at http://www.iup. physik.uni-bremen.de/sciatran.

(C) 2012 Published by Elsevier Ltd. on behalf of COSPAR

Keywords: Radiative transfer; Ocean-atmosphere coupling

\section{Introduction}

The radiative transfer (RT) model SCIATRAN was originally developed to analyse measurements performed by the hyperspectral instrument SCIAMACHY (SCanning Imaging $A$ bsorption Spectro $M$ eter for $A$ tmospheric

$C H \operatorname{artograph} Y$ ) operating in the spectral range from 240 to $2400 \mathrm{~nm}$ onboard ENVISAT (Bovensmann et al., 1999; Gottwald, 2006). SCIATRAN is a comprehensive software package (Rozanov et al., 2002; Rozanov et al., $2005,2008)$ for the modeling of radiative transfer processes

\footnotetext{
* Corresponding author. Address: Institute of Environmental Physics, University of Bremen, FB 1, P.O. Box 330440, 28334 Bremen, Germany. Tel.: +49 42121862081 .

E-mail address: blum@iup.physik.uni-bremen.de (M. Blum).

${ }^{1}$ (alt.: Otto Hahn Allee 1, 28359 Bremen), Germany.
}

in the terrestrial atmosphere in the spectral range from ultraviolet to the thermal infrared $(0.18-40 \mu \mathrm{m})$ including multiple scattering processes, polarization, and thermal emission. The software allows to consider all significant radiative transfer processes such as Rayleigh scattering, scattering by aerosol and cloud particles, and absorption by numerous gaseous components in the vertically inhomogeneous atmosphere bounded by the reflecting surface. The reflecting properties of a surface are described by the bidirectional reflection function including Fresnel reflection of the flat and wind roughened ocean-atmosphere interface. The developed software package along with detailed User's Guide are freely distributed at http://www.iup.physik.unibremen.de/sciatran. It contains databases of all important atmospheric and surface parameters as well as many defaults mode which significantly facilitate the usage of SCIATRAN for non-experts in radiative transfer users. 
Although the developed software can be used to solve numerous forward and inverse problems of the atmospheric optics, it does not allow to model e.g. radiation field in the ocean and, in particular, the water leaving radiation containing important information about numerous ocean optical parameters (e.g. Vountas et al. (2007), Bracher et al. (2009)). Furthermore, the accuracy of trace gas and aerosol retrievals over oceanic sites can be improved including the interaction of radiative processes in the atmosphere and ocean in the corresponding RT model.

For this reason, the software package SCIATRAN was extended, to account for the radiative transfer within the water and the interaction of radiative processes in the atmosphere and ocean. Although a number of coupled ocean-atmosphere RT models including polarization effects have been recently published (Bulgarelli et al., 1999; Fell and Fischer, 2001; He et al., 2010; Jin et al., 2006; Ota et al., 2010; Zhai et al., 2010), only the COART model (Jin et al., 2006) permits an online usage by providing a set of input parameters; however, the source code is not available, only an interface is given on the website http:// snowdog.larc.nasa.gov/jin/rtnote.html. To our knowledge, the SCIATRAN model is the only free available software to calculate radiative transfer in a coupled ocean-atmosphere system.

The main goals of this paper are

- To describe the optical properties of natural waters implemented in the code;

- To discuss modifications in the formulation of the RT equation and boundary conditions in the case of the coupled ocean-atmosphere system;

- To present a new iterative technique that is employed to solve boundary value problem in the coupled oceanatmosphere RT model;

- to demonstrate validation results of the extended SCIATRAN version.

Taking into account that the atmospheric radiative transfer of the SCIATRAN software was successfully validated (see e.g. Kokhanovsky et al. (2010)), we restrict ourselves here to the validation of the oceanic radiative transfer. The validation is performed through intercomparisons with benchmark results and predictions of other RT models as well as through comparisons with MERIS (MEdium Resolution Imaging Spectrometer) (Bezy et al., 2000) spectra measured over oceanic sites.

\section{Basic principles of ocean optics}

The principles of Ocean Colour are characterized in Fig. 1. Solar radiation is absorbed and scattered by atmospheric constituents, and reflected and refracted at the airwater interface.

Within water, the transmitted solar radiation is absorbed and scattered, and after interaction with water constituents, the solar radiaton reenters the atmosphere.

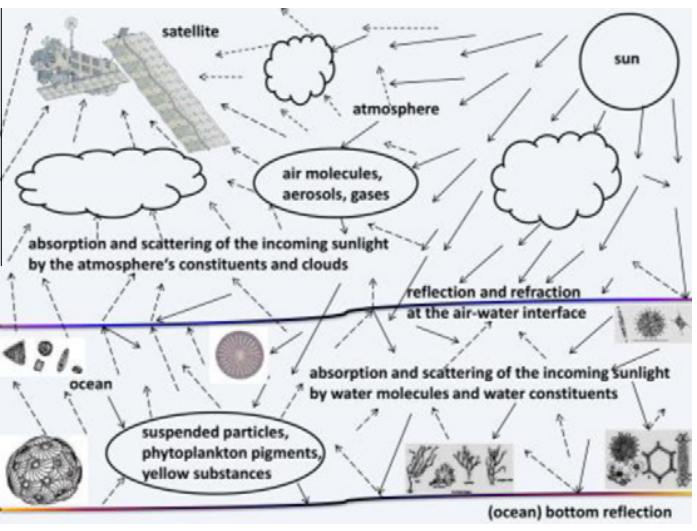

Fig. 1. Principles of ocean colour.

Finally, before detection at an instrument, the water leaving radiance interacts with atmospheric constituents again.

In order to analyse the radiative processes within water, adequate knowledge of the optical properties of water itself and of its constituents, where the main optically active substances besides water molecules are CDOM (Coloured Dissolved Organic Matter), phytoplankton, and suspended particles, is required. One thereby distinguishes between IOPs (Inherent $O$ ptical Properties), which are only depending on the medium itself, and thus independent on the surrounding lightfield, and AOPs (Apparent Optical Properties), which are depending on the IOPs as well as on the surrounding elctromagnetic radiation field. Typical IOP parameters are the absorption coefficient $a$, the volume scattering function $\beta$, and the scattering coefficient $b$, whereas e.g. reflectance and transmittance are AOPs. To deduce the information about the particular oceanic constituent from the measured data, accurate knowledge of the optical parameters of oceanic species and the behaviour of electromagnetic radiation in the water medium is essential.

\section{Radiative transfer in the coupled ocean-atmosphere system}

The radiative transfer in the atmosphere and ocean will be considered in the framework of the standard BVP (Boundary Value Problem) (Chandrasekhar, 1950):

$\mu \frac{\partial I_{\mathrm{tot}}(\tau, \Omega)}{\partial \tau}=-I_{\mathrm{tot}}(\tau, \Omega)+J_{\mathrm{tot}}(\tau, \Omega)$,

$I_{\text {tot }}(0, \Omega)=\pi \delta\left(\mu-\mu_{0}\right) \delta\left(\varphi-\varphi_{0}\right), \quad \mu>0$,

$I_{\text {tot }}\left(\tau_{0}, \Omega\right)=\mathcal{R} I_{\text {tot }}\left(\tau_{0}, \Omega^{\prime}\right), \quad \mu<0$.

Here, $\tau \in\left[0, \tau_{0}\right]$ is the optical depth changing from 0 at the top of the plane-parallel medium to $\tau_{0}$ at the bottom, the variable $\Omega:=\{\mu, \varphi\}$ describes the set of variables $\mu \in[-1,1]$ and $\varphi \in[0,2 \pi], \mu$ is the cosine of the polar angle $\vartheta$ as measured from the positive $\tau$-axis (negative $z$-axis) and $\varphi$ is the azimuthal angle, $I_{\text {tot }}(\tau, \Omega)$ is the total intensity (or radiance) at the optical depth $\tau$ in the direction $\Omega, J_{\text {tot }}(\tau, \Omega)$ is the multiple scattering source function, and $\mathcal{R}$ is a linear 
integral operator. The multiple scattering source function and linear integral operator $\mathcal{R}$ are given as follows:

$$
\begin{aligned}
& J_{\text {tot }}(\tau, \Omega)=\frac{\omega(\tau)}{4 \pi} \int_{4 \pi} P\left(\tau, \Omega, \Omega^{\prime}\right) I_{\mathrm{tot}}\left(\tau, \Omega^{\prime}\right) d \Omega^{\prime}, \\
& \mathcal{R}=\frac{1}{\pi} \int_{0}^{2 \pi} d \varphi^{\prime} \int_{0}^{1} d \mu^{\prime} \mu^{\prime} R\left(\Omega, \Omega^{\prime}\right) \otimes,
\end{aligned}
$$

where $\omega(\tau)$ is the single scattering albedo (scattering coefficient divided by extinction coefficient), $P\left(\tau, \Omega, \Omega^{\prime}\right)$ is the phase function describing angular scattering properties of the medium, and $R\left(\Omega, \Omega^{\prime}\right)$ determines angular reflection properties of the underlying surface, symbol $\otimes$ is used to denote an integral operator rather than a finite integral.

The UBC (Upper Boundary Condition) given by Eq. (2) describes the unidirectional $\left(\mu_{0}, \varphi_{0}\right)$ solar light beam at the top of atmosphere, $\delta\left(\mu-\mu_{0}\right)$ and $\delta\left(\varphi-\varphi_{0}\right)$ are the Dirac delta functions, $\mu_{0}$ and $\varphi_{0}$ are the cosines of the solar zenith angle and solar azimuthal angle, respectively. The solar zenith angle is defined as an angle between positive direction of $z$-axis and the direction to the sun. The $x$-axis of basic Cartesian coordinate system is chosen so that its direction is opposite to the direction to the sun. Therefore, the azimuthal angle of the solar beam equal to zero $\left(\varphi_{0}=0\right)$. It follows from Eq. (2) that the extraterrestrial solar flux at an unit horizontal area is equal to $\pi \mu_{0}$.

The LBC (Lower Boundary Condition) given by Eq. (3) defines the bidirectional reflection of radiation at the surface. In particular, in the case of Lambertian reflection the integral operator $\mathcal{R}$ results in

$$
\mathcal{R}_{\mathrm{L}}=\frac{A}{\pi} \int_{0}^{2 \pi} d \varphi^{\prime} \int_{0}^{1} d \mu^{\prime} \mu^{\prime} \otimes,
$$

where $A$ is the Lambertian surface albedo.

$\downarrow$ Formulating the RT equation along with boundary conditions given by Eqs. (1)-(3), we have restricted ourselves with the scalar case i.e., polarization is not included. The thermal emission is not included also because it is of minor importance for the RT processes in the ocean.

The formulated BVP for the total intensity includes generalized functions in the form of Dirac $\delta$-functions (see Eq. (2)). It is known that solutions of such equations contain the generalized functions as well. The standard approach to eliminate the generalized function in the solution of the RT equation is to separate the total intensity into direct and diffuse component and to formulate the RT equation for the diffuse component only (Chandrasekhar, 1950). In this case the total intensity is represented as follows (Chandrasekhar, 1950):

$I_{\text {tot }}(\tau, \Omega)=I(\tau, \Omega)+D(\tau, \Omega)$,

where $I(\tau, \Omega)$ and $D(\tau, \Omega)$ are the diffuse and direct components of the total intensity, respectively.

Substituting $I_{\text {tot }}(\tau, \hat{\Omega})$ given by Eq. (7) into Eq. (1) and introducing the multiple and single scattering source functions as follows:
$J_{m}(\tau, \Omega)=\frac{\omega(\tau)}{4 \pi} \int_{4 \pi} P\left(\tau, \Omega, \Omega^{\prime}\right) I\left(\tau, \Omega^{\prime}\right) d \Omega^{\prime}$,

$J_{s}(\tau, \Omega)=\frac{\omega(\tau)}{4 \pi} \int_{4 \pi} P\left(\tau, \Omega, \Omega^{\prime}\right) D\left(\tau, \Omega^{\prime}\right) d \Omega^{\prime}$,

we obtain the following RT equation and boundary conditions for the diffuse component:

$\mu \frac{\partial I(\tau, \Omega)}{\partial \tau}=-I(\tau, \Omega)+J_{m}(\tau, \Omega)+J_{s}(\tau, \Omega)$,

$I(0, \Omega)=0, \quad \mu>0$

$I\left(\tau_{0}, \Omega\right)=\mathcal{R} D\left(\tau_{0}, \Omega^{\prime}\right)+\mathcal{R} I\left(\tau_{0}, \Omega^{\prime}\right), \quad \mu<0$,

where the integral operator $\mathcal{R}$ is given by Eq. (5). Eqs. (10)-(12) describe BVP for the intensity of the diffuse radiation field.

Employing appropriate boundary conditions and expressions for the direct component $D(\tau, \Omega)$, the formulated BVP can be used to model RT processes in the atmosphere and ocean. These issues will be considered in the three following subsections.

\subsection{Uncoupled atmospheric and oceanic radiative transfer models}

Ignoring the coupling, the corresponding BVP can be formulated for both ocean and atmosphere independently. It can be seen from Eqs. (9) and (12) that the single scattering source function $J_{s}(\tau, \Omega)$ and LBC depend on the direct component $D(\tau, \Omega)$. Therefore, to describe radiative transfer in the atmosphere it will be used the following representation of the direct solar component:

$$
\begin{aligned}
D_{\mathrm{a}}(\tau, \Omega)= & \pi \delta\left(\mu-\mu_{0}\right) \delta\left(\varphi-\varphi_{0}\right) e^{-\tau / \mu_{0}} \\
& +\pi \delta\left(\mu+\mu_{0}\right) \delta\left(\varphi-\varphi_{0}\right) R_{\mathrm{F}}\left(\mu_{0}\right) e^{-\left(2 \tau_{a}-\tau\right) / \mu_{0}},
\end{aligned}
$$

where $R_{\mathrm{F}}\left(\mu_{0}\right)$ is the Fresnel reflection coefficient of the water surface and $\tau_{a}$ is the optical thickness of the entire atmosphere. The first term in this equation describes the attenuation of the direct solar radiation by the atmosphere at the optical depth $\tau$ and the second one is used if the Fresnel reflection from the absolute flat water surface is accounted for. This term describes the upward direct solar radiation at the optical depth $\tau$ reflected by the water surface and attenuated by the atmosphere.

The direct solar component in the ocean at the optical depth $\tau$ is used as follows:

$D_{\mathrm{o}}(\tau, \Omega)=\pi \delta\left(\mu-\mu_{0}^{\prime}\right) \delta\left(\varphi-\varphi_{0}\right) \frac{\mu_{0}}{\mu_{0}^{\prime}} T_{\mathrm{F}}\left(\mu_{0}\right) e^{-\tau / \mu}$.

Here $T_{\mathrm{F}}\left(\mu_{0}\right)$ is the Fresnel transmission coefficient of the air-water interface, $\tau$ is the optical depth in the ocean, and $\mu_{0}^{\prime}$ is the cosine of the solar angle in the ocean defined according to Snell law (Born and Wolf, 1964) as $\mu_{0}^{\prime}=\sqrt{1-\left(1-\mu_{0}^{2}\right) / n^{2}}$, where $n$ is the real part of the water refractive index. We assume throughout this paper that the refractive index of the air is equal to 1 . The multiplier $\mu_{0} / \mu_{0}^{\prime}$ is introduced in the expression (14) to ensure the energy 
conservation of the direct solar radiation just above and just below the ocean surface.

Substituting expressions (13) and (14) into Eqs. (9) and (12), we obtain the single scattering source function and LBC in the atmosphere and ocean, respectively. The UBC for the atmosphere is given always by Eq. (11) which manifests that there is no diffuse radiation incoming in the atmosphere from the top. In contrast to the atmosphere at the top of ocean there is an jump of refractive index. This leads to the Fresnel reflection of the outgoing radiation at the top of the ocean. In particular, the part of energy will be reflected back into ocean. To take this into account one needs to reformulate the upper boundary condition for the intensity in the ocean. To this end we write in the case of the wind-roughened ocean surface

$I(0, \Omega)=\mathcal{R}_{\mathrm{w}} I\left(0, \Omega^{\prime}\right), \quad \mu>0$,

where $\boldsymbol{\mathcal { R }}_{\mathrm{w}}$ denotes a linear integral operator

$\mathcal{R}_{\mathrm{W}}=\frac{1}{\pi} \int_{0}^{2 \pi} d \varphi^{\prime} \int_{-1}^{0} d \mu^{\prime} \mu^{\prime} R_{\mathrm{W}}\left(\Omega, \Omega^{\prime}\right) \otimes$,

$R_{\mathrm{w}}\left(\Omega, \Omega^{\prime}\right)$ determines the angular reflection properties of the upper ocean boundary and $I(0, \Omega)$ describes the intensity of the radiation reflected from the ocean-atmosphere interface back to the ocean. In the case of the flat ocean surface the linear integral operator $\mathcal{R}_{\mathrm{w}}$ should be replaced by the Fresnel reflection coefficient $R_{F}\left(\mu^{\prime}\right)$.

The boundary conditions and single scattering source functions corresponding to the uncoupled atmospheric and oceanic RT model are summarized in the left and right columns of Table 1 , respectively.

It is worth to notice that:

- Single scattering albedo, phase function, and the optical thickness in the left and right columns of Table 1 describe the optical parameters of the atmosphere and ocean, respectively;

- Fresnel reflection $R_{\mathrm{F}}(\mu)$ and transmission $T_{\mathrm{F}}(\mu)$ coefficients of the flat ocean surface are used as given e.g. by Born and Wolf (1964);

- Fresnel reflection and transmission of the wind-roughened air-water interface was implemented in SCIATRAN according to Nakajima and Tanaka (1983) including shadowing effects and Gaussian distribution of wave slopes;

- The water-leaving radiation $I_{\mathrm{WL}}(\Omega)$ is used according to the modified Gordon approximation (Anikonov and Ermolaev, 1977; Gordon, 1973; Kokhanovsky and Sokoletsky, 2006);

- The uncoupled atmospheric RT model is implemented already in the software package SCIATRAN;

- Only Lambertian reflection of the ocean bottom is implemented in the current version;

- The typical example of the uncoupled oceanic RT model is the widely used in the ocean optics community HydroLight model (Mobley and Sundman, 2008a; Mobley and Sundman, 2008b).

\subsection{Coupled ocean-atmosphere radiative transfer model}

The coupled ocean-atmosphere RT model has the same upper boundary condition in the atmosphere and lower boundary condition in the ocean as uncoupled one. However, LBC in the atmosphere and UBC in the ocean have to be corrected to properly account for interaction of radiative processes in the atmosphere and ocean. In particular, a part of energy is transmitted from the ocean through the air-water interface into the atmosphere. To take this into account, LBC for the atmosphere given e.g. in the case of wind-roughened ocean surface in the left panel of Table 1 should be rewritten as follows:

$$
\begin{aligned}
I\left(\tau_{0}, \Omega\right)= & R\left(\Omega, \Omega_{0}\right) \mu_{0} e^{-\tau_{0} / \mu_{0}}+\mathcal{R} I\left(\tau_{0}, \Omega^{\prime}\right)+\mathcal{T}_{\mathrm{wa}} I\left(\tau_{0}^{+}, \Omega^{\prime}\right), \\
& \mu<0,
\end{aligned}
$$

where $I\left(\tau_{0}^{+}, \Omega^{\prime}\right)$ is the intensity of radiation field just below the air-water interface. The transmission operator $\mathcal{T}_{\text {wa }}$ is given by

$\mathcal{T}_{\text {wa }}=\int_{0}^{2 \pi} d \varphi^{\prime} \int_{-1}^{0} d \mu^{\prime} T_{\text {wa }}\left(\Omega, \Omega^{\prime}\right) \otimes$,

where $T_{\text {wa }}\left(\Omega, \Omega^{\prime}\right)$ denotes the angular transmission properties of the air-water interface for illumination from below. The last term in Eq. (17) describes the so called water leaving radiation which is introduced here instead of approxi-

Table 1

$\mathrm{UBC}, \mathrm{LBC}$, and $J_{\mathrm{s}}$ of the uncoupled radiative transfer model.

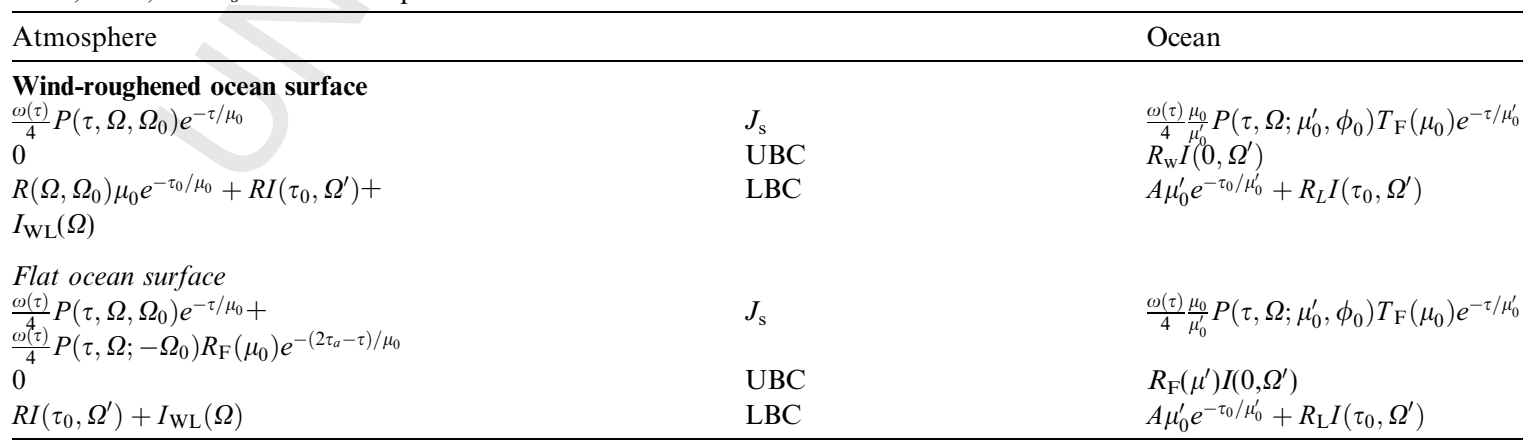


mation, $I_{\mathrm{WL}}(\Omega)$, used in the case of the uncoupled atmospheric radiative transfer model.

The atmosphere above the ocean attenuates the direct solar radiation transmitted into ocean. There is also the diffuse radiation illuminating the ocean surface from above. Denoting the optical thickness of the atmosphere as $\tau_{a}$, the direct solar radiation in the ocean is obtained as follows:

$\mathbf{D}_{\mathrm{o}}(\tau, \Omega)=\pi \delta\left(\mu-\mu_{0}^{\prime}\right) \delta\left(\varphi-\varphi_{0}\right) \frac{\mu_{0}}{\mu_{0}^{\prime}} T_{\mathrm{F}}\left(\mu_{0}\right) e^{-\tau_{\alpha} / \mu_{0}} e^{-\tau / \mu}$,

where $\tau$ is the optical depth counted from the top of the ocean. The upper boundary condition in the ocean has to be rewritten also to account for the diffuse radiation transmitted from the atmosphere into ocean. This results in

$I(0, \Omega)=\mathcal{R}_{\mathrm{w}} I\left(0, \Omega^{\prime}\right),+\mathcal{T}_{\mathrm{aw}} I\left(\tau_{0}^{-}, \Omega^{\prime}\right), \quad \mu>0$,

where $I\left(\tau_{0}^{-}, \Omega^{\prime}\right)$ is the intensity of radiation field just above the air-water interface. The transmission operator $\mathcal{T}_{\text {aw }}$ is given in the form analogical to Eq. (18), where $T_{\mathrm{wa}}\left(\Omega, \Omega^{\prime}\right)$ should be replaced by $T_{\text {aw }}\left(\Omega, \Omega^{\prime}\right)$ which describes the angular transmission properties of the air-water interface for illumination from above.

The single scattering source functions and corresponding boundary conditions for coupled ocean-atmosphere model are summarized in Table 2: we note that

- The direct solar radiation in the ocean is used ignoring the wind-roughness, i.e., for the flat air-water interface. Therefore, the single scattering source function in the right panel of Table 2 is the same for wind-roughened and flat ocean surface.

- Integral operators $\mathcal{T}_{\text {wa }}$ and $T_{\text {aw }}$ describing the transmission of the radiation across air-water interface are implemented according to Nakajima and Tanaka (1983).

\subsection{Solution of the boundary value problem}

To solve formulated above BVP we employ the Fourier analysis to separate the zenith and azimuthal dependence of the intensity (Siewert, 1981; Siewert, 1982; Siewert, 2000) and the discrete ordinates technique
(Chandrasekhar, 1950; Schulz et al., 1999; Schulz and Stamnes, 2000; Siewert, 2000; Stamnes et al., 1988; Thomas and Stamnes, 1999) for the reduction of integro-differential equations to the system of ordinary differential equations. In particular, the expansion of the intensity and phase function into Fourier series leads to the formulation of independent system of equations for each Fourier harmonics of the intensity. To obtain the solution of RT equation for the $m$-th Fourier harmonic the discrete ordinates method is used. According to this technique, the radiation field is divided into $N$ up-welling and $N$ down-welling streams, producing the intensity pairs $I_{-}(\tau)$ and $I_{+}(\tau)$ in the discrete directions $\pm \mu_{i}$, where $\mu_{i}$ are quadrature points of the double-Gauss scheme (see e.g. Thomas and Stamnes (1999) for details) adopted in SCIATRAN. Considering the radiative transfer in the atmosphere, the Gaussianquadrature points and weights are the same in all atmospheric layers, but it is not the case for the coupled ocean-atmosphere medium because the refraction at the interface of the atmosphere and ocean occurs. For the flat sea surface, the incident radiance with the zenith angle between $0^{\circ}-90^{\circ}$ in the atmosphere transmits in the ocean in a cone (so called Fresnel cone) with the maximum zenith angle less than the critical angle $\left(\sim 48.3^{\circ}\right)$. Therefore, the number of Gaussian-quadrature points in the ocean must be larger than the number in the atmosphere to properly account for the radiative transfer in the region of total reflection (i.e. outside the Fresnel cone). To this end, the so called coupled underwater quadrature points method as used by Jin et al. (2006) has been implemented in SCIATRAN. This method uses two sets of quadrature points, one corresponds to the refracted directions in the atmosphere and the other covers the region outside the Fresnel cone. The detailed discussion of the coupled quadrature points method is given e.g. by He et al. (2010).

Having defined the Gaussian-quadrature points and applying a quadrature formula to replace all integrals over the direction cosine by finite sums in the RT equation, one arrives at a system of coupled first order ordinary linear differential equations in the optical depth $\tau$.

Comparing the boundary conditions of the uncoupled and coupled RT model given in Tables 1 and 2, respectively, one can see that UBC for the ocean contains the

Table 2

UBC, LBC, and $J_{\mathrm{s}}$ for coupled ocean-atmosphere model.

\begin{tabular}{|c|c|c|}
\hline Atmosphere & & Ocean \\
\hline $\begin{array}{l}\text { Wind-roughened ocean surface } \\
\frac{\omega(\tau)}{4} P\left(\tau, \Omega, \Omega_{0}\right) e^{-\tau / \mu_{0}} \\
0 \\
R\left(\Omega, \Omega_{0}\right) \mu_{0} e^{-\tau_{0} / \mu_{0}}+R I\left(\tau_{0}, \Omega^{\prime}\right)+ \\
T_{\mathrm{wa}} I\left(\tau_{0}^{+}, \Omega^{\prime}\right)\end{array}$ & $\begin{array}{l}J_{\mathrm{s}} \\
\mathrm{UBC} \\
\mathrm{LBC}\end{array}$ & 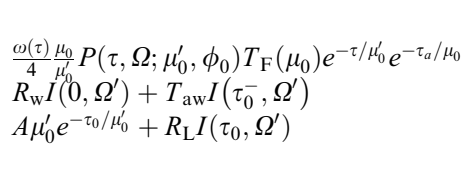 \\
\hline $\begin{array}{l}\text { Flat ocean surface } \\
\frac{\omega(\tau)}{\frac{\omega}{4}} P\left(\tau, \Omega, \Omega_{0}\right) e^{-\tau / \mu_{0}}+ \\
\frac{0}{4} P\left(\tau, \Omega ;-\mu_{0}, \phi_{0}\right) R_{\mathrm{F}}\left(\mu_{0}\right) e^{-\left(2 \tau_{a}-\tau\right) / \mu_{0}} \\
R I\left(\tau_{0}, \Omega^{\prime}\right)+T_{\mathrm{F}}\left(\mu^{\prime}\right) I\left(\tau_{0}^{+}, \Omega^{\prime}\right)\end{array}$ & $\begin{array}{l}J_{\mathrm{s}} \\
\mathrm{UBC} \\
\mathrm{LBC}\end{array}$ & 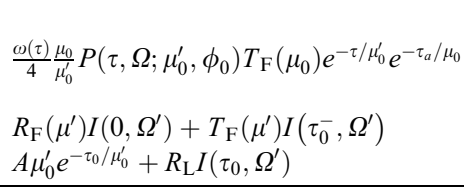 \\
\hline
\end{tabular}


contribution of the transmitted across the air-water interface intensity, $I\left(\tau_{0}^{-}, \Omega^{\prime}\right)$, which is defined just above the ocean surface, i.e. in the atmosphere. The same is hold for LBC in atmosphere. This contains the contribution of the transmitted across the air-water interface intensity, $I\left(\tau_{0}^{+}, \Omega^{\prime}\right)$, which is defined just below the ocean surface, i.e., in the ocean. Thus, the solution of BVP in the atmosphere depends on the solution in the ocean and vice versa. Therefore, to solve BVP for the coupled ocean-atmosphere RT model, an iterative technique has been employed. To illustrate this, the solution of BVP in the atmosphere and ocean is written in the following symbolic form:

$I_{\mathrm{a}}^{\mathrm{n}}(\tau, \Omega)=L_{\mathrm{a}}^{-1} S_{\mathrm{a}}(\tau, \Omega)+L_{\mathrm{a}}^{-1}\left[\mathcal{T}_{\mathrm{wa}} I_{\mathrm{w}}^{\mathrm{n}-1}\left(0, \Omega^{\prime}\right)\right]$,

$I_{\mathrm{w}}^{\mathrm{n}}(\tau, \Omega)=L_{\mathrm{w}}^{-1} S_{\mathrm{w}}(\tau, \Omega)+L_{\mathrm{w}}^{-1}\left[\mathcal{T}_{\mathrm{aw}} I_{\mathrm{a}}^{\mathrm{n}}\left(\tau_{0}, \Omega^{\prime}\right)\right]$ where $L$ is the forward RT operator which comprises all operations with the intensity including boundary conditions, $S\left(\tau_{2} \Omega\right)$ is the right-hand side of the forward RT equation written in generalized form (see Rozanov and Rozanov (2007) for details), $L^{-1}$ is an inverse operator, $\mathrm{n}$ is the iteration number, subscripts " $a$ " and "w" denote the corresponding parameters in the atmosphere and ocean, respectively, $I_{\mathrm{w}}\left(0, \Omega^{\prime}\right)$ and $I_{\mathrm{a}}\left(\tau_{0_{\Omega}} \Omega^{\prime}\right)$ denote the intensity just below and just above the air-water interface, respectively. The iteration process is started from the solution of RTE in the atmosphere ignoring the water-leaving radiation, i.e. setting $I_{\mathrm{w}}^{0}\left(0, \Omega^{\prime}\right)=0$ in Eq. (21). The solution in the atmosphere is obtained as $I_{\mathrm{a}}^{1}(\tau, \Omega)$. The solution of RTE in ocean is then found as follows:

$I_{\mathrm{w}}^{1}(\tau, \Omega)=L_{\mathrm{w}}^{-1} S_{\mathrm{w}}(\tau, \Omega)+L_{\mathrm{w}}^{-1}\left[\mathcal{T}_{\mathrm{aw}} I_{\mathrm{a}}^{1}\left(\tau_{0}, \Omega^{\prime}\right)\right]$

Table 3

Optical properties of natural waters implemented in SCIATRAN.

Total spectral absorption coefficient of seawater $\boldsymbol{a}(\boldsymbol{\lambda}, \boldsymbol{C})$ : $a(\lambda, C)=a_{w}(\lambda)+a_{C}(\lambda)+a_{p}(\lambda)$

$a_{w}(\lambda)$

$a_{C}(\lambda)$

$a_{p}(\lambda)$
Absorption coefficient of pure water in $\left[\mathrm{m}^{-1}\right]$ (Pope and Fry, 1997).

Chlorophyll related absorption coefficient:

$0.06 \cdot A_{c}(\lambda) \cdot C^{0.65}\left[\mathrm{~m}^{-1}\right]$

Pigment (dissolved organic matter or CDOM) absorption coefficient:

$0.2 \cdot\left[a_{w}\left(\lambda_{0}\right)+0.06 \cdot C^{0.65}\right] \cdot e^{-S\left(\lambda-\lambda_{0}\right)}\left[m^{-1}\right]$

$\left(a_{C} \& a_{p}\right.$ according to Morel and Maritorena (2001))

for $\lambda_{0}=440 \mathrm{~nm}$,

$S=0.014(\mathrm{~nm})^{-1}$, chlorophyll concentration $C[$ $\mathrm{mg} \cdot \mathrm{m}^{-3}$,

and $A_{c}(\lambda)$ with $A_{c}\left(\lambda_{0}\right)=1$ (Prieur and Sathyendranath, 1981).

Angular scattering coefficient of seawater $\beta(\lambda, \Theta)$ :

$\boldsymbol{\beta}(\lambda, \boldsymbol{\Theta})=\boldsymbol{\beta}_{w}(\lambda, \boldsymbol{\Theta})+\boldsymbol{\beta}_{p}(\lambda, \Theta)\left[\frac{1}{\mathbf{m} \cdot \mathbf{r}}\right]$

$\beta_{w}(\lambda, \Theta)$

Angular scattering coefficient or volume scattering function of

pure water:

$\boldsymbol{\beta}_{w}\left(\lambda, 90^{\circ}\right)\left(1+\frac{1-\delta}{1+\delta} \cos ^{2} \Theta\right)\left[\frac{1}{\mathrm{~m} \cdot \mathrm{sr}}\right]$.

$\beta_{w}\left(\lambda, 90^{\circ}\right)$

Volume scattering function of pure water at $90^{\circ}$ scattering angle:

$\frac{2 \pi^{2}}{\lambda^{4} B_{T}} k T_{a} n^{2}\left(\frac{\partial n}{\partial P}\right)_{T}^{2}\left(\frac{6+6 \delta}{6-7 \delta}\right)\left[\frac{1}{\mathrm{~m} \cdot \mathrm{ss}}\right]$.

$\boldsymbol{b}_{w}(\lambda)$

$\boldsymbol{\beta}_{p}(\lambda, \Theta)$

Implemented models of $\beta_{w}\left(\lambda, 90{ }^{\circ}\right)$ and $b_{w}(\lambda)$ :

Morel (1974)

Shifrin (1988)

Buiteveld et al. (1994)

Implemented models of $\boldsymbol{\beta}_{p}(\lambda, \Theta)$ :

Petzold (1972)

Kopelevich (1983) $\frac{8 \pi}{3} \beta_{w}\left(\lambda, 90^{\circ}\right)\left(\frac{2+\delta}{1+\delta}\right)\left[\frac{1}{m}\right]$.

Angular scattering coefficient of particulate matter.

$\beta_{w}\left(\lambda, 90^{\circ}\right)=2.18 \cdot\left(\frac{450}{\zeta 5}\right)^{4.32} \cdot 10^{-4}\left[\frac{1}{\mathrm{~m} \cdot \mathrm{sr}}\right]$

$b_{w}(\lambda)=3.50 \cdot\left(\frac{450}{\lambda}\right)^{4.32} \cdot 10^{-3}\left[\frac{1}{m}\right]$

$b_{w}(\lambda)=3.50 \cdot\left(\frac{450}{\lambda}\right) \cdot 10^{-3}\left[\frac{1}{m}\right]$
$\beta_{w}\left(\lambda, 90^{\circ}\right)=0.93 \cdot\left(\frac{546}{4.17}\right)^{-17} \cdot 10^{-4}\left[\frac{1}{\mathrm{~m} \cdot \mathrm{sr}}\right]$

$b_{w}(\lambda)=1.49 \cdot\left(\frac{546}{\lambda}\right)^{4.1^{\gamma}} \cdot 10^{-3}\left[\frac{1}{m}\right]$

Inserting provided set of formulas and values (Table 4) into $\boldsymbol{\beta}_{w}\left(\lambda, 9^{\circ}\right)$.

Values from experiments which were presented by Haltrin (2006)

$\beta_{p}(\lambda, \Theta)=v_{s} \beta_{s}(\Theta) \cdot\left(\frac{550}{\lambda}\right)^{1.7}+v_{l} \beta_{l}(\Theta) \cdot\left(\frac{550}{\lambda}\right)^{0.3}\left[\frac{1}{\mathrm{~m} \cdot \mathrm{sr}}\right]$ for volume concentrations $v_{s}$ of small and $v_{l}$ of large particles in

$\left[\mathrm{cm}^{3} \cdot \mathrm{m}^{-3}\right]$.
Total scattering coefficient of pure water: 
The iteration process will be stopped if the difference between values of the water-leaving intensity in the two subsequent iterations is less than the required criteria.

\subsection{Optical properties of natural waters}

The inherent optical properties specify the optical properties of natural waters in a form suited to the needs of radiative transfer theory. In the first line it is the spectral absorption coefficient, spectral attenuation (or extinction) coefficient and spectral volume scattering function (or phase function). All IOPs implemented in SCIATRAN are listed in Table 3. We note that

- The approximation of pure water angular scattering coefficient given by Morel (1974) and Shifrin (1988) refers to measurements at $T=20^{\circ} \mathrm{C}$ and depolarization ratio $\delta$ equal to 0.09 at atmospheric pressure.

- The salinity adjustment factor is set to $[1+0.3 S / 37]$ according to Morel (1974) and Shifrin (1988), where $S$ is salinity.

- Functions $\left.\beta_{s_{\perp}} \Theta\right)$ and $\beta_{l}(\Theta)$ in the Kopelevich model (Kopelevich, 1983) are used in the tabular form.

- The volume concentrations $v_{s}$ and $v_{l}$ of particles in the Kopelevich model can be converted into the conventional mass concentrations $C_{s}$ and $C_{l}$ by $C_{s}=\rho_{s} v_{s}, C_{l}=\rho_{l} v_{l}$, where $\rho_{s}=2 \mathrm{~g} \cdot \mathrm{cm}^{-3}$ and $\rho_{l}=1 \mathrm{~g} \cdot \mathrm{cm}^{-3}$ are the average density of small and large particles, respectively.

It follows from Table 3 that calculation of some IOPs requires the specific parameters of the water constituents such as pressure, temperature, salinity, chlorophyll and particulate matter concentration profiles, and so on. Thus, the SCIATRAN data base was filled up with profile data of depth distributions, as well as with absorption coefficients of pure water and specific absorption coefficients of chlorophyll.

\section{Validation of the extended model}

The coupled ocean-atmosphere RT model implemented in the software package SCIATRAN has been validated using two approaches. First, we have compared results obtained with SCIATRAN to the different test problems (Mobley et al., 1993), and then the calculated reflectances at the top of atmosphere were compared to the MERIS measured reflectances. The measurements performed with the MERIS instrument have been selected for this comparison because the spatial resolution of MERIS instrument $\left(\sim 1 \times 1 \mathrm{~km}^{2}\right)$ fully resolved the peculiarity of the BOUSSOLE station. It is worth to notice that the BOUSSOLE station is already a case- 1 water site, although it is located only $59 \mathrm{~km}$ off the coast. Therefore the high spatial resolution of a satellite instrument is required to avoid possible contribution of case-2 water features.

A brief description of results obtained is given in two following subsections.

\subsection{Comparison to other model data}

The predictions of SCIATRAN were compared with those of a number of other models for selected well-defined test cases, covering specific aspects of the radiative transfer in the ocean-atmosphere system as presented by Mobley et al. (1993). Although seven test problems were defined in the cited above paper we have restricted ourselves to four following:

1. Optically semi-infinite and vertically homogeneous ocean.

- Refractive index of water $n=1.34$,

- Flat ocean-atmosphere interface,

- $60^{\circ}$ solar zenith angle and $\mathrm{E}_{0}=1 \mathrm{Wm}^{-2} \mathrm{~nm}^{-1}$ incident solar irradiance,

- Black sky,

- Pure water scattering described by Rayleigh phase function,

- Single scattering albedo values $\omega_{0}=0.2$ and $\omega_{0}=0.9$.

2. The same as 1 but more realistic Petzold phase function is used instead of Rayleigh one.

3 . The same as 2 but for the vertically stratified ocean.

4. The same as 2 but including atmospheric effects.

The following radiative quantities were involved in the comparison study:

$$
\begin{aligned}
& E_{d}(\tau)=\mu_{0} E_{0} T_{\mathrm{F}}\left(\mu_{0}\right) e^{-\tau / \mu_{0}^{\prime}}+2 \pi \int_{0}^{1} I^{0}(\tau, \mu) \mu d \mu, \\
& E_{0 u}(\tau)=2 \pi \int_{-1}^{0} I^{0}(\tau, \mu) d \mu, L_{u}(\tau)=I^{0}(\tau,-1),
\end{aligned}
$$

where $\mathrm{E}_{d}(\tau), \mathrm{E}_{0 u}(\tau)$ and $\mathrm{L}_{u}(\tau)$ are the total downward irradiance, upward scalar irradiance and upward nadir radiance, respectively, at the optical depth $\tau, I^{0}(\tau, \mu)$ is the azimuthally averaged intensity, $\mu_{0}$ and $\mu_{0}^{\prime}$ are cosines of the solar zenith angle in the atmosphere and ocean, respectively, $T_{\mathrm{F}}\left(\mu_{0}\right)$ is the Fresnel transmission coefficient.

These radiative quantities were calculated for test problems listed above employing seven RT models (see Mobley et al. (1993) for details). The discussion of these models is out scope of this paper because it will be used here the average values and standard deviations only which characterize the variability of results obtained with involved in the comparison study RT codes. Recently the solution of the first three test problems has been obtained also by other RT models. In particular, these test problems were solved employing matrix operator method (MOMO, Fell and Fischer, 2001), finite-element method (FEM, Bulgarelli et al., 1999), and invariant embedding method (demo version of HydroLight 5.1, Mobley and Sundman, 2008a; Mobley and Sundman, 2008b). This motivates our choice of three first test problems for inter-comparisons. Let us consider all results obtained. Calculated values of $\mathrm{E}_{d}, \mathrm{E}_{0 u}$, and $\mathrm{L}_{u}$ 
alone with average values and standard deviations given by Mobley et al. (1993) are summarized in Tables 5 and 6 for test problems 1 and 2. Results are given at three optical depths $(\tau=1,5,10)$ and two single scattering albedo $\left(\omega_{0}=0.2,0.9\right)$. It follows that HydroLight, SCIATRAN, and MOMO results are very close to each other and stand within the standard deviations given by Mobley et al. (1993). We recall that the standard deviations indicate the variability of results obtained with codes involved in the comparison study by Mobley et al. (1993). It can be seen also that the relative deviations increase with the decreasing of the single scattering albedo. It can be explained due to the fact that the increasing of absorption (decreasing of SSA) leads to the significant decreasing of irradiance and especially of upward nadir radiance. Results obtained for the test problem 3 are summarized in Fig. 2 and Table 7. In this test problem the single scattering albedo is assumed to be strongly dependent on the depth (see Mobley et al., 1993 for further details). It can be seen from Fig. 2 that for this more complicated test problem the SCIATRAN predictions are within the error bars for all depth under consideration. In particular, it follows from middle panel of Fig. 2 that for the upward scalar irradiance $\left(\mathrm{E}_{0 u}\right)$ at the geometrical depth $60 \mathrm{~m}$ only SCIATRAN result is within the error bars (Mobley et al., 1993). Except for this specific depth, the FEM model results (Bulgarelli et al., 1999) are within the error bars also.

Table 4

General constants and parameters of the volume scattering function according to Buiteveld et al. (1994).

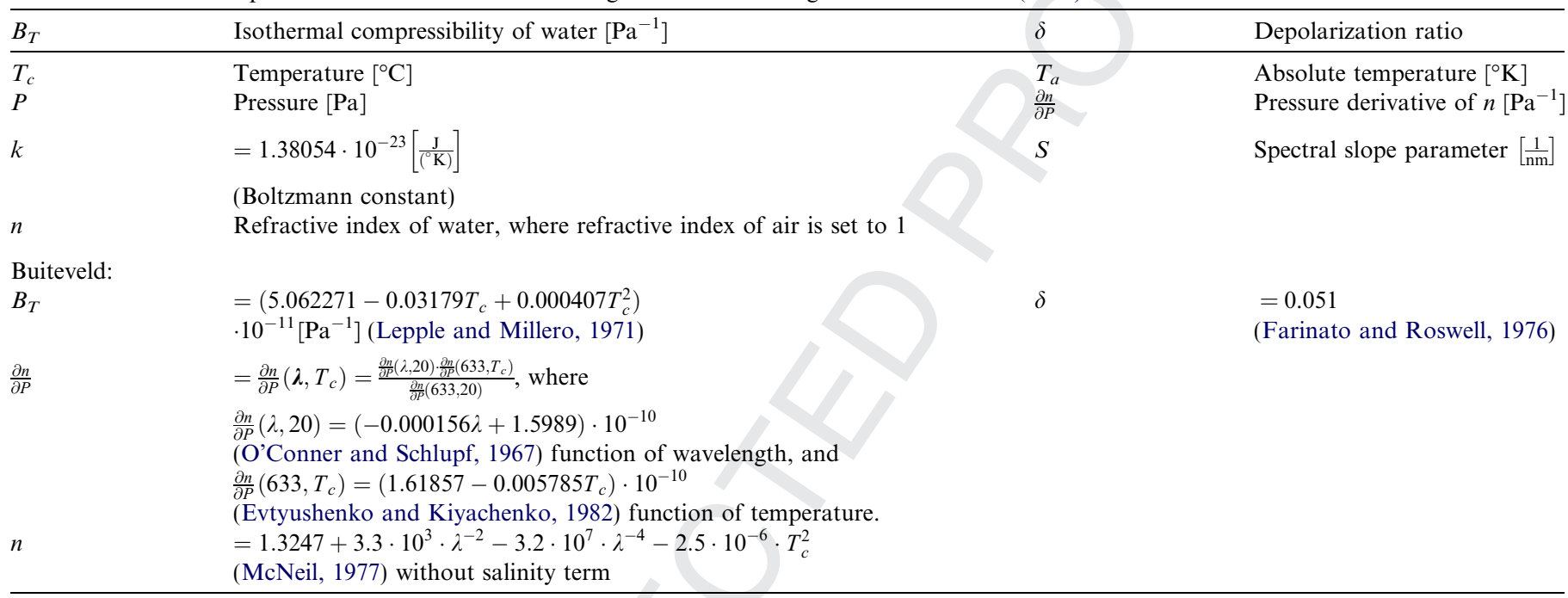

Table 5

Results for optically semi-infinite and vertically homogeneous ocean with the Rayleigh volume scattering function (test problem 1).

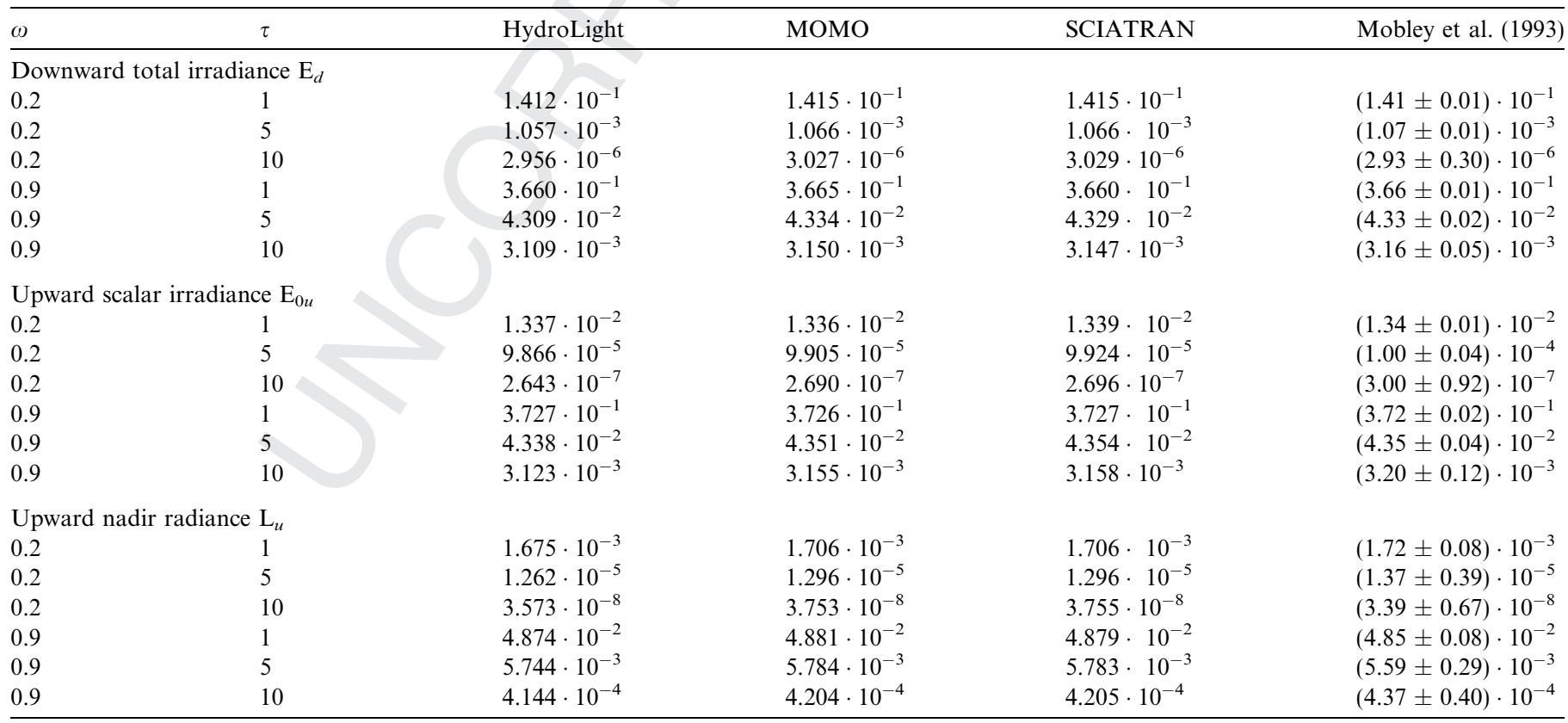


Table 6

Results for optically semi-infinite and vertically homogeneous ocean with the Petzold volume scattering function (test problem 2).

\begin{tabular}{|c|c|c|c|c|c|}
\hline$\omega$ & $\tau$ & HydroLight & MOMO & SCIATRAN & Mobley et al. (1993) \\
\hline \multicolumn{6}{|c|}{ Downward total irradiance $\mathrm{E}_{d}$} \\
\hline 0.2 & 1 & $1.617 \cdot 10^{-1}$ & $1.622 \cdot 10^{-1}$ & $1.621 \cdot 10^{-1}$ & $(1.62 \pm 0.01) \cdot 10^{-1}$ \\
\hline 0.2 & 5 & $2.267 \cdot 10^{-3}$ & $2.283 \cdot 10^{-3}$ & $2.277 \cdot 10^{-3}$ & $(2.27 \pm 0.01) \cdot 10^{-3}$ \\
\hline 0.2 & 10 & $1.314 \cdot 10^{-5}$ & $1.309 \cdot 10^{-5}$ & $1.312 \cdot 10^{-5}$ & $(1.30 \pm 0.07) \cdot 10^{-5}$ \\
\hline 0.9 & 1 & $4.129 \cdot 10^{-1}$ & $4.137 \cdot 10^{-1}$ & $4.135 \cdot 10^{-1}$ & $(4.13 \pm 0.01) \cdot 10^{-1}$ \\
\hline 0.9 & 5 & $1.856 \cdot 10^{-1}$ & $1.884 \cdot 10^{-1}$ & $1.868 \cdot 10^{-1}$ & $(1.87 \pm 0.01) \cdot 10^{-1}$ \\
\hline 0.9 & 10 & $6.752 \cdot 10^{-2}$ & $6.942 \cdot 10^{-2}$ & $6.832 \cdot 10^{-2}$ & $(6.85 \pm 0.07) \cdot 10^{-2}$ \\
\hline \multicolumn{6}{|c|}{ Upward scalar irradiance $\mathrm{E}_{0 u}$} \\
\hline 0.2 & 1 & $9.651 \cdot 10^{-4}$ & $9.541 \cdot 10^{-4}$ & $9.894 \cdot 10^{-4}$ & $(9.66 \pm 0.22) \cdot 10^{-4}$ \\
\hline 0.2 & 5 & $1.333 \cdot 10^{-5}$ & $1.325 \cdot 10^{-5}$ & $1.370 \cdot 10^{-5}$ & $(1.37 \pm 0.09) \cdot 10^{-5}$ \\
\hline 0.2 & 10 & $6.963 \cdot 10^{-8}$ & $6.905 \cdot 10^{-8}$ & $7.143 \cdot 10^{-8}$ & $(7.28 \pm 1.36) \cdot 10^{-8}$ \\
\hline 0.9 & 1 & $9.470 \cdot 10^{-2}$ & $9.192 \cdot 10^{-2}$ & $9.470 \cdot 10^{-2}$ & $(9.31 \pm 0.20) \cdot 10^{-2}$ \\
\hline 0.9 & 5 & $4.673 \cdot 10^{-2}$ & $4.574 \cdot 10^{-2}$ & $4.679 \cdot 10^{-2}$ & $(4.63 \pm 0.08) \cdot 10^{-2}$ \\
\hline 0.9 & 10 & $1.641 \cdot 10^{-2}$ & $1.635 \cdot 10^{-2}$ & $1.656 \cdot 10^{-2}$ & $(1.65 \pm 0.03) \cdot 10^{-2}$ \\
\hline \multicolumn{6}{|c|}{ Upward nadir radiance $\mathrm{L}_{u}$} \\
\hline 0.2 & 1 & $5.575 \cdot 10^{-5}$ & $5.546 \cdot 10^{-5}$ & $5.832 \cdot 10^{-5}$ & $(5.47 \pm 0.33) \cdot 10^{-5}$ \\
\hline 0.2 & 5 & $7.885 \cdot 10^{-7}$ & $7.873 \cdot 10^{-7}$ & $8.142 \cdot 10^{-7}$ & $(6.24 \pm 2.22) \cdot 10^{-7}$ \\
\hline 0.2 & 10 & $4.574 \cdot 10^{-9}$ & $4.525 \cdot 10^{-9}$ & $4.667 \cdot 10^{-9}$ & $(4.02 \pm 1.00) \cdot 10^{-9}$ \\
\hline 0.9 & 1 & $6.981 \cdot 10^{-3}$ & $6.783 \cdot 10^{-3}$ & $7.001 \cdot 10^{-3}$ & $(6.99 \pm 0.44) \cdot 10^{-3}$ \\
\hline 0.9 & 5 & $3.161 \cdot 10^{-3}$ & $3.117 \cdot 10^{-3}$ & $3.186 \cdot 10^{-3}$ & $(3.26 \pm 0.18) \cdot 10^{-3}$ \\
\hline 0.9 & 10 & $1.138 \cdot 10^{-3}$ & $1.138 \cdot 10^{-3}$ & $1.154 \cdot 10^{-3}$ & $(1.21 \pm 0.13) \cdot 10^{-3}$ \\
\hline
\end{tabular}
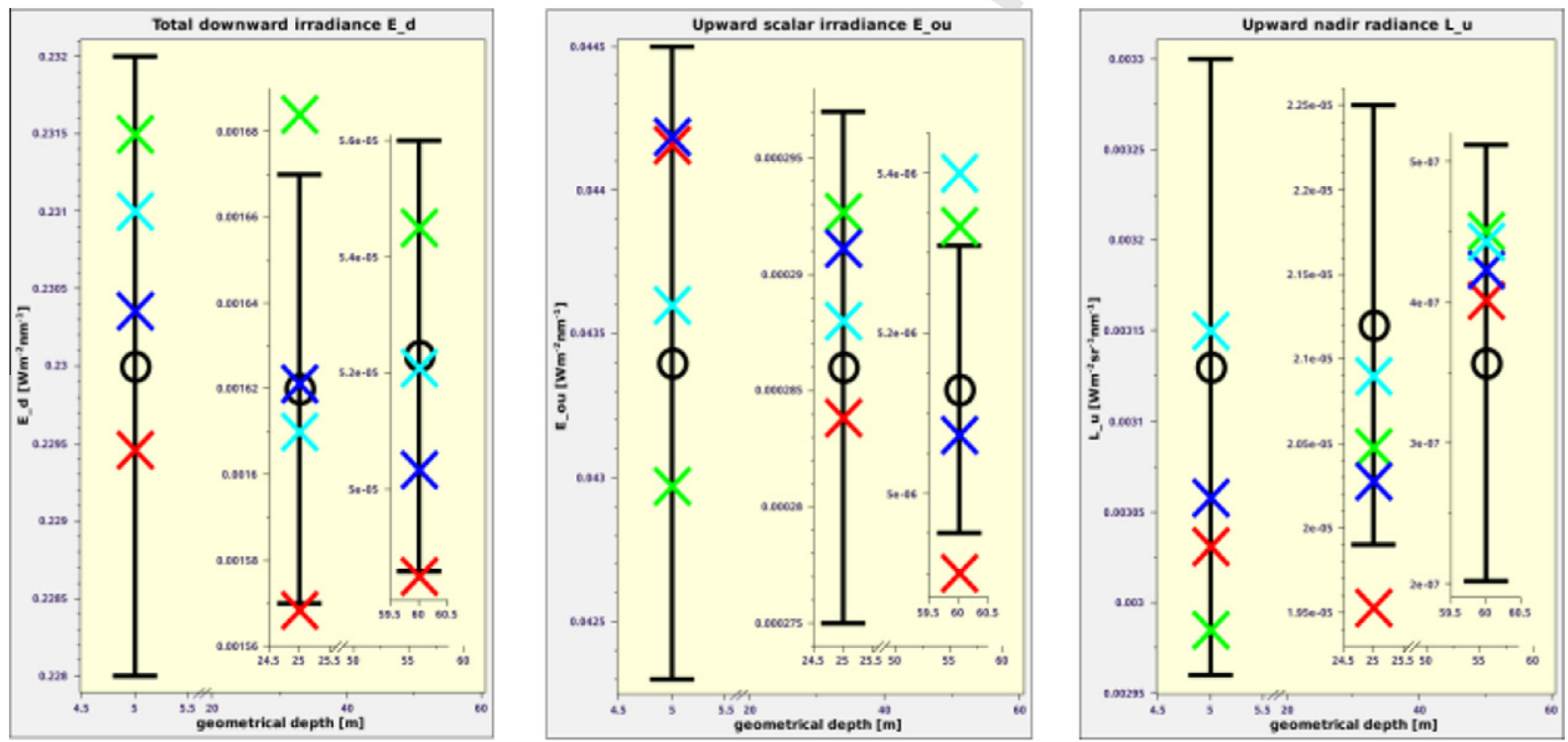

Fig. 2. Results for the test problem 3 obtained with SCIATRAN, $(\times)$, HydroLight 5.1 (DemoVersion, $\times$ ), MOMO ( $\times$, Fell and Fischer (2001)), and FEM $(\times$, Bulgarelli et al. (1999)) models in relation to the average value (०) and standard deviation $(\top \perp)$ as given in Mobley et al. (1993).

The test problem 4 was selected to validate SCIATRAN in the case of coupling of radiative transfer processes in the ocean-atmosphere system. In contrast to test problems 1-3 it includes atmospheric effects. The sky is no longer black as in previous test problems but has the radiance distribution that describes the atmospheric scattering and absorption effects. The atmosphere was characterized by the Rayleigh and aerosol optical thicknesses equal to 0.145 and 0.264 , respectively. Because no more detailed specification of atmospheric aerosol was given, we have reproduced this scenario with SCIATRAN using different values of asymmetry factor and single scattering albedo of aerosol particles. The simulations show, however, that the influence of these parameters on the radiation field in water is rather small especially for upward scalar irradiance and nadir radiance. Fig. 3 shows results obtained employing SCIATRAN to the test problem 4 setting asymmetry aerosol factor to 0.7 and the single scattering albedo to 1 and 0.9. It follows that in both cases the obtained results are within error bars.

Concluding we can state that SCIATRAN can successfully reproduce all considered test scenarios. In particular, 
Table 7

Results for the vertically inhomogeneous ocean (test problem 3).

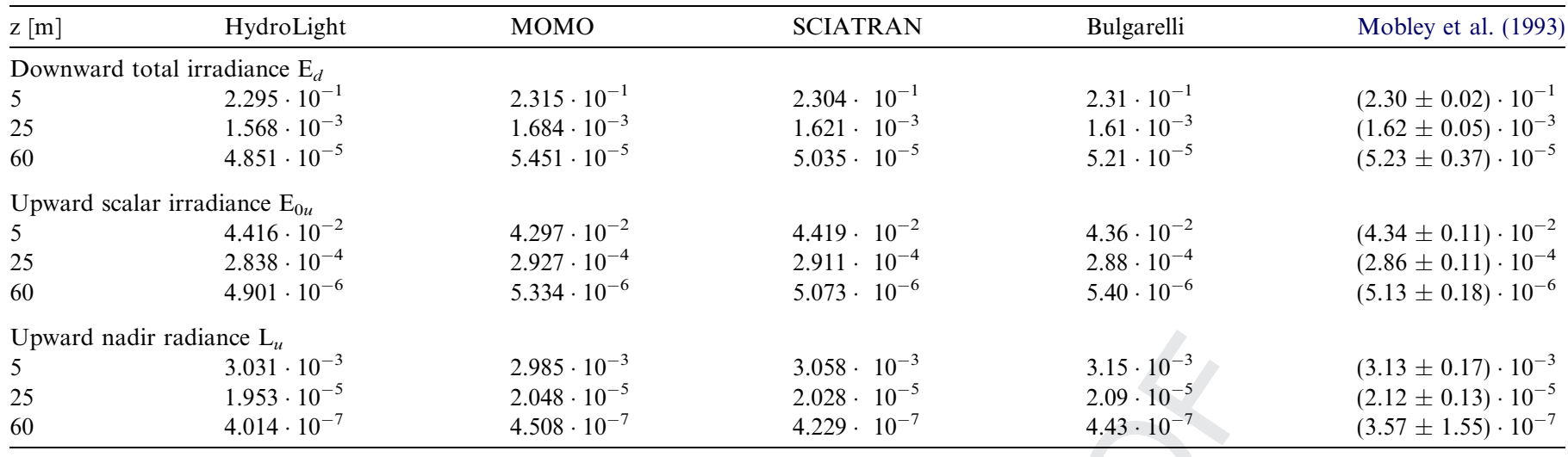
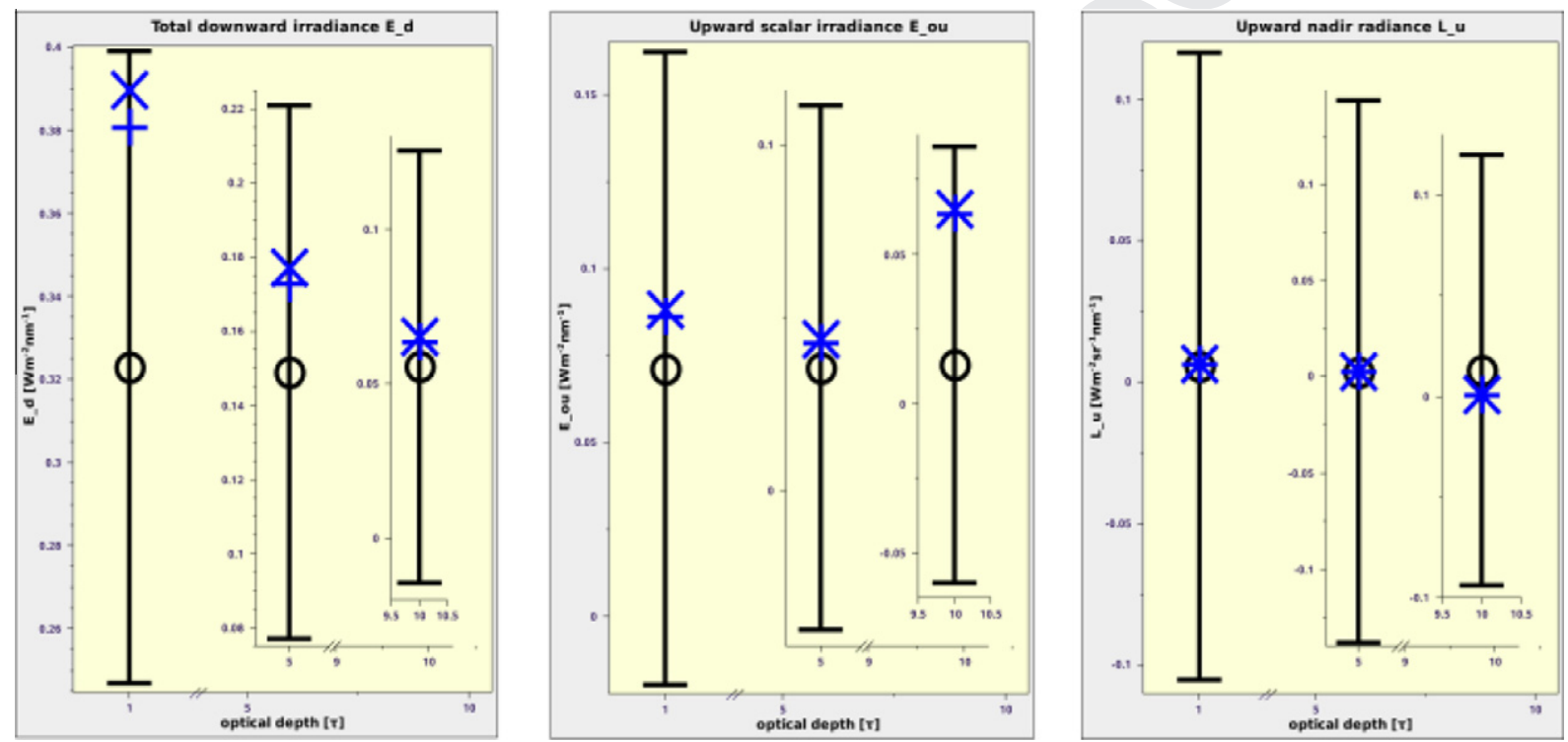

Fig. 3. Results for the test problem 4 obtained with the SCIATRAN model setting asymmetry factor and single scattering albedo of aerosol to 0.7 and 1.0 $(\times)$, and to 0.7 and $0.9(+)$ in relation to the average value $(\circ)$ and error bar $(\top \perp)$ as given in Mobley et al. (1993).

test problems 1-3 demonstrate that the implementation of uncoupled oceanic RT model in the software package SCIATRAN is correct. The solution of the test problem 4 shows that in the considered case the impact of the coupling on the light field within water is not too much.

\subsection{Comparison to MERIS measurements}

Comparisons of spectra calculated with SCIATRAN and measured by MERIS were performed for the reflectance at the top of atmosphere (reftoa). The reflectance is defined as follows:

$R(\vartheta, \varphi, \lambda)=\frac{\pi I(\vartheta, \varphi, \lambda)}{E_{0}(\lambda) \cos \vartheta_{0}}$

where $I(\vartheta, \varphi, \lambda)$ is the radiance at given zenith $\vartheta$ and azimuthal $\varphi$ angles, $E_{0}(\lambda)$ is the extraterrestrial solar spectral irradiance, $\lambda$ is the wavelength, and $\vartheta_{0}$ denotes the sun zenith angle. We have used the reflectance for comparisons of model and experimental data because it does not contain any additional systematical errors caused by employing atmospheric correction techniques that are usually used to obtain water-leaving radiance.

To calculate $R(\vartheta, \varphi, \lambda)$ one needs to define all relevant atmospheric and oceanic parameters. In particular, the following parameters are required:

- MERIS data have been used to define the observation geometry, the solar zenith angle, the atmospheric pressure, the concentrations of $\mathrm{H}_{2} \mathrm{O}$ and $\mathrm{O}_{3}$, the aerosol optical thickness at 550 and $865 \mathrm{~nm}$, and the geographical position of measurement points;

- AERONET data (http://aeronet.gsfc.nasa.gov/) have been used to obtain the aerosol phase function, the aerosol extinction coefficient, and the aerosol single scattering albedo at 440, 675, 870, and $1020 \mathrm{~nm}$; 
- ocean parameters such as temperature, salinity, chlorophyll concentration, and concentrations of small and large particles at different depths were obtained from the BOUSSOLE data (BOUSSOLE boy at $43.6^{\circ} \mathrm{E}$, $7.8^{\circ} \mathrm{N}$; see Antoine et al., 2008);

- the reflection and transmission properties of the ocean surface were defined by the Gaussian surface slope PDF (including shadowing effects) and the mean square slope as given by Cox and Munk (1954), Cox and Munk (1954), the pure seawater and hydrosol volume scattering functions were used according to Buiteveld et al. (1994) and Kopelevich (1983) models, respectively.

In order to perform comparisons, MERIS data suitable for the comparison with model calculations were matched to co-located AERONET and BOUSSOLE measurement sites. Moreover, the time difference between measurements performed by the MERIS instrument, AERONET, and BOUSSOLE data was kept as small as possible, since time differences between the different measurements up to 10 hours occured. There are some additional criteria by choosing the MERIS data for the comparison, e.g. cloudy scenes and the observation geometry near to the solar glint have to be avoided. Taking into account all of the above mentioned criteria, 20 MERIS matches at different seasons in 2003-2006 have been obtained. Ten spectra still need further investigation, since the results show errors which might be caused by various factors, such as high surface roughness, the wind speed being too high, dim air, or sun glint.

The reflectance at the top of atmosphere was calculated employing the uncoupled atmospheric and coupled oceanatmosphere RT models implemented in the SCIATRAN software (see Sections 3.1 and 3.2, respectively). For simplification reasons they will be referred to in following as COA and unCOA models. In the later case the information about atmospheric and ocean surface parameters was used in the same way as for the coupled model, but the water-leaving reflectance was calculated according to the modified Gordon approximation (Gordon, 1973). The uncoupled model does not consider the radiative transfer processes in the ocean and it does not utilize any information about vertical profiles of the oceanic parameters such as the concentrations of chlorophyll, large and small particles. Therefore, maximal differences between results obtained employing the coupled and uncoupled models are expected for cases of strong vertical inhomogeneities of these oceanic parameters.

\begin{tabular}{|l|l|l|l|}
\hline matter & surface conc. $(\mathbf{O ~ m})$ & max. conc. at () $\mathbf{m}$ & vertical gradient per $\mathbf{m}$ \\
\hline TChla & $0.250 \mathrm{mg} / \mathrm{m}^{3}$ & $0.250 \mathrm{mg} / \mathrm{m}^{3}$ at $05 \mathrm{~m}$ & $0.0000 \mathrm{mg} / \mathrm{m}^{3}$ \\
\hline small particles & $0.057 \mathrm{mg} / \mathrm{m}^{3}$ & $0.058 \mathrm{mg} / \mathrm{m}^{3}$ at $10 \mathrm{~m}$ & $0.0001 \mathrm{mg} / \mathrm{m}^{3}$ \\
\hline large particles & $0.221 \mathrm{mg} / \mathrm{m}^{3}$ & $0.221 \mathrm{mg} / \mathrm{m}^{3}$ at $05 \mathrm{~m}$ & $0.0000 \mathrm{mg} / \mathrm{m}^{3}$ \\
\hline
\end{tabular}
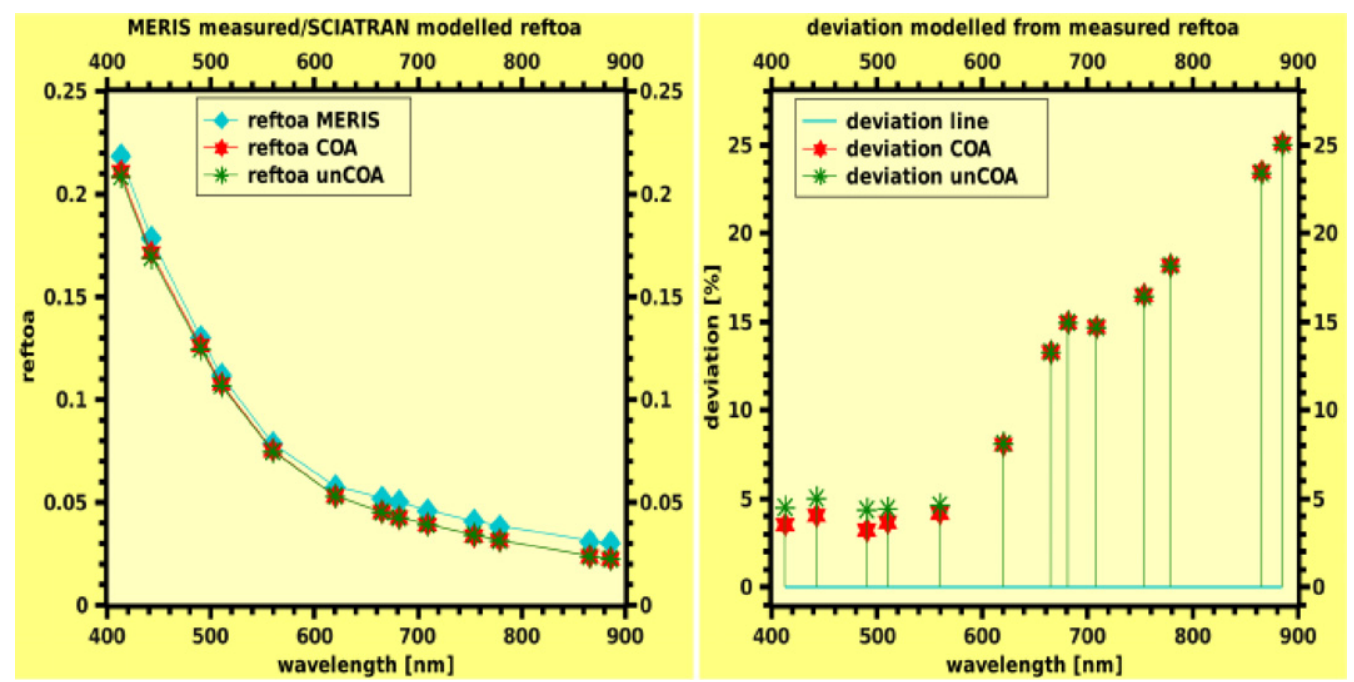

Fig. 4. Comparison of reftoa measured by MERIS and calculated with coupled (COA) and uncoupled (unCOA) SCIATRAN models at the BOUSSOLE station (left) and its deviation (right) for February 23, 2005. The mean deviation between measured and modeled with COA and unCOA reflectances are $11.8 \%$ and $12.1 \%$, respectively. In the upper panel the settings of the oceanic parameters for these calculations are given. 
The preliminary analysis of all results obtained shows that indeed in the case of almost vertically homogeneous ocean both models produce very similar results. In particular, Fig. 4 shows, as an example, the comparison of modelled reflectance with the MERIS measurement performed in February 23, 2005, in the case of low varying with depth chlorophyll and particulate matter concentrations. Both models describe in this case the measured reflectance spectra with a similar accuracy.

Figs. 5 and 6 show the comparison of modelled reflectances with the MERIS measurements performed in April 5, 2003, and in July 31, 2004, respectively. According to the BOUSSOLE data, the vertical profiles of chlorophyll concentration show very similar vertical gradients $\left(\sim 0.027 \mathrm{mg} \cdot \mathrm{m}^{-3} / \mathrm{m}\right)$ for both days, but the chlorophyll concentration at the ocean surface was more than three times larger in April than in July. Comparing results presented in Figs. 5 and 6, we can conclude that for both days the coupled model shows better coincidence with the MERIS spectra in the spectral range relevant to the chlorophyll absorption $(400-560 \mathrm{~nm})$, where pure seawater absorption is low. Furthermore, Fig. 7 (October 5, 2006) demonstrates the impact of the vertical gradient of the chlorophyll concentration on the performance of the coupled and uncoupled models. Using the BOUSSOLE data, the vertical gradient of the chlorophyll concentration was estimated as $\sim 0.019 \mathrm{mg} \cdot \mathrm{m}^{-3} / \mathrm{m}$, which is $\sim 1.4$ times smaller than the gradient in April and July (see Figs. 5 and 6, respectively). It follows from Fig. 7 that decreasing of the vertical gradient leads to the improvement of the performance of the uncoupled model.

Considering further results presented in Figs. 4-7, we can state that

- the performance of the coupled and uncoupled RT models is approximately the same for wavelengths greater than $\sim 600 \mathrm{~nm}$;

- the difference between modeled and measured spectra increases in the spectral range $600-900 \mathrm{~nm}$.

Simular performance of the coupled and uncoupled RT models for wavelengths greater than $\sim 600 \mathrm{~nm}$ can be explained due to the fact that absorption of pure seawater increases with the increasing of wavelength. Indeed, the absorption coefficient of pure water is $\sim 0.06 \mathrm{~m}^{-1}$ at $550 \mathrm{~nm}$, enhances to $\sim 0.34 \mathrm{~m}^{-1}$ at $650 \mathrm{~nm}$ and reaches $\sim 2.6 \mathrm{~m}^{-1}$ at $750 \mathrm{~nm}$ (see e.g. Haltrin, 2006). The increasing of the absorption coefficient leads to the decreasing of the photon penetration depth and, therefore, mitigates effects caused by the vertical inhomogeneity.

The enhancement of differences between measured and modelled spectra in the spectral range $600-900 \mathrm{~nm}$ can be
703

704

705

706

\begin{tabular}{|l|l|l|l|}
\hline matter & surface conc. $(0 \mathrm{~m})$ & max. conc. at () $\mathrm{m}$ & vertical gradient per $\mathrm{m}$ \\
\hline TChla & $0.718 \mathrm{mg} / \mathrm{m}^{3}$ & $1.262 \mathrm{mg} / \mathrm{m}^{3}$ at $20 \mathrm{~m}$ & $0.0272 \mathrm{mg} / \mathrm{m}^{3}$ \\
\hline small particles & $0.080 \mathrm{mg} / \mathrm{m}^{3}$ & $0.164 \mathrm{mg} / \mathrm{m}^{3}$ at $20 \mathrm{~m}$ & $0.0042 \mathrm{mg} / \mathrm{m}^{3}$ \\
\hline large particles & $0.732 \mathrm{mg} / \mathrm{m}^{3}$ & $1.284 \mathrm{mg} / \mathrm{m}^{3}$ at $50 \mathrm{~m}$ & $0.0110 \mathrm{mg} / \mathrm{m}^{3}$ \\
\hline
\end{tabular}
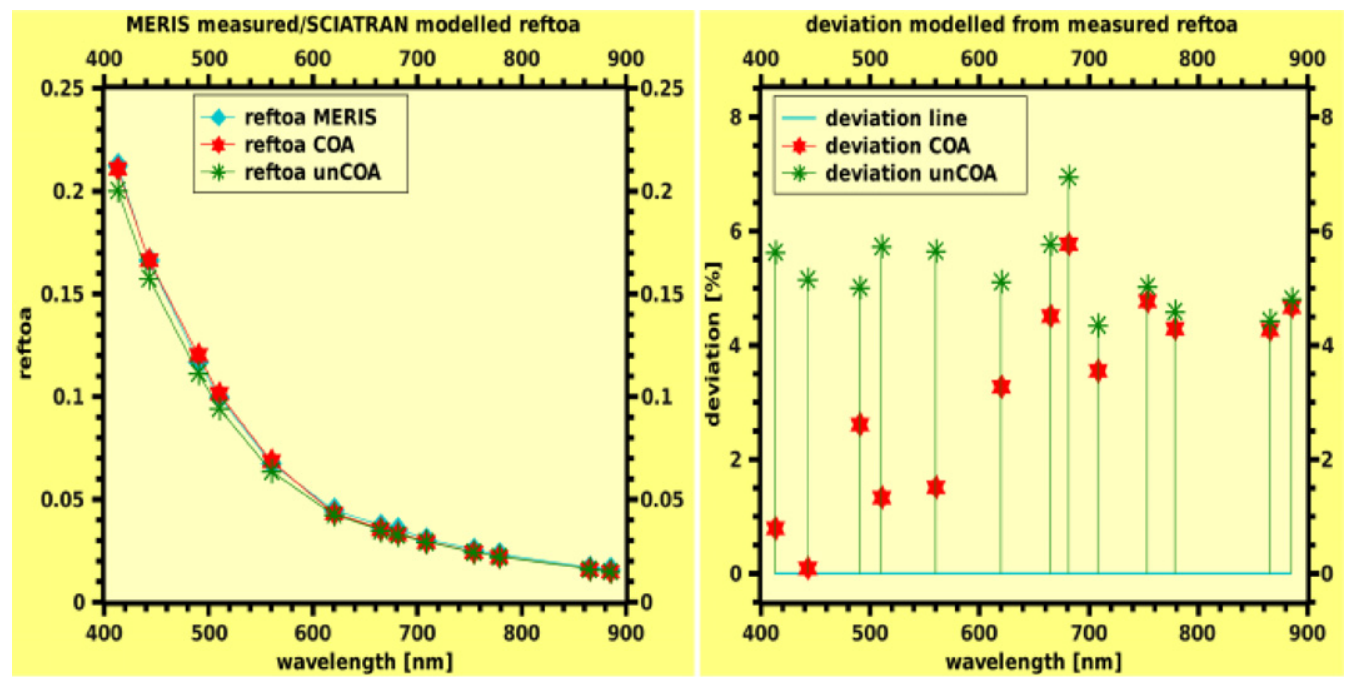

Fig. 5. The same as in Fig. 4, but for April 5, 2003. The mean deviation of COA and unCOA is 3.2\% and 5.3\%, respectively. 


\begin{tabular}{|l|l|l|l|}
\hline matter & surface conc. $(0 \mathrm{~m})$ & max. conc. at () $\mathrm{m}$ & vertical gradient per $\mathbf{m}$ \\
\hline TChla & $0.201 \mathrm{mg} / \mathrm{m}^{3}$ & $1.587 \mathrm{mg} / \mathrm{m}^{3}$ at $50 \mathrm{~m}$ & $0.0277 \mathrm{mg} / \mathrm{m}^{3}$ \\
\hline small particles & $0.045 \mathrm{mg} / \mathrm{m}^{3}$ & $0.257 \mathrm{mg} / \mathrm{m}^{3}$ at $40 \mathrm{~m}$ & $0.0053 \mathrm{mg} / \mathrm{m}^{3}$ \\
\hline large particles & $0.165 \mathrm{mg} / \mathrm{m}^{3}$ & $1.704 \mathrm{mg} / \mathrm{m}^{3}$ at $50 \mathrm{~m}$ & $0.0308 \mathrm{mg} / \mathrm{m}^{3}$ \\
\hline
\end{tabular}
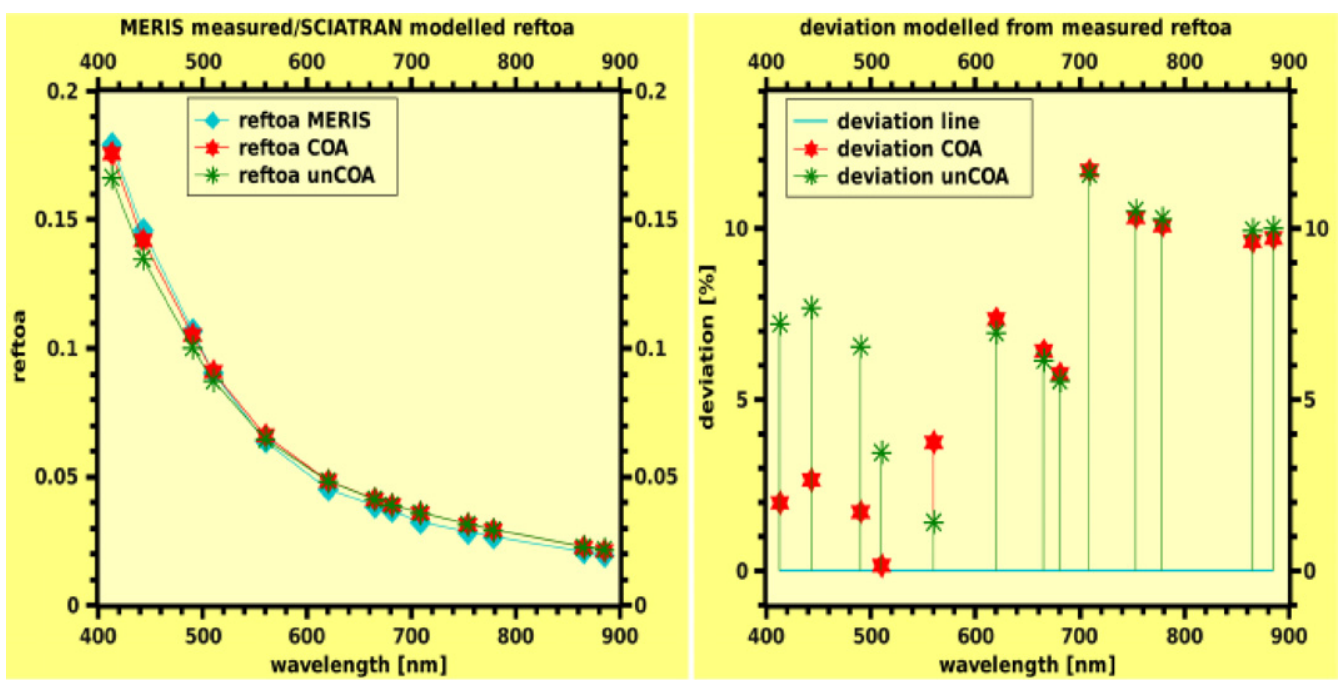

Fig. 6. The same as in Fig. 4, but for July 31, 2004. The mean deviation of COA and unCOA is $6.2 \%$ and $7.5 \%$, respectively.

explained by the ignoring of inelastic scattering processes such as vibrational Raman scattering and fluorescence in the current version of our RT model. However, a more realistic reason of this difference can be the lack of exact information on atmospheric aerosol parameters.

\section{Conclusion}

We have discussed the theoretical background of radiative transfer processes and inherent optical parameters of the natural water implemented in the extended version of the software package SCIATRAN. The extended SCIATRAN versions 3.1 and greater allow users to account for not only the radiative processes within the atmosphere but also within the ocean including they interaction.

Taking into account that the atmospheric radiative transfer of the SCIATRAN software has been successfully validated (Kokhanovsky et al., 2010), we have presented here the validation of the oceanic radiative transfer. Comparisons of SCIATRAN results to the predictions of other RT models used to solve selected well-defined test problems, covering specific aspects of the radiative transfer in the ocean-atmosphere system (Mobley et al., 1993), have demonstrated good performance of the extended SCIATRAN version to calculate the radiative transfer within water. In order to establish that all physical processes are properly incorporated to describe radiative processes in the coupled ocean-atmosphere system we have presented comparisons of the model predictions with measurements performed by MERIS instrument. Comparisons show good agreement between measured and modeled reflectances in the spectral range $400-550 \mathrm{~nm}$ where the coupling effects are significant. This demonstrates that the extended SCIATRAN version can be employed to model satellite measurements of the reflected radiation performed over oceanic sites properly accounting for the vertical distribution of oceanic parameters. The contribution of the water-leaving radiance into the final detected signal is at maximum approximately $10 \%$ to the backscattered radiance measured by the satellite sensor. Therefore, the accuracy of the RT modeling at the top of the atmosphere radiation should not exceed more than one to two percent. Also for atmospheric retrievals of trace gases accuracy within a few percent is needed. This also requires that $\mathrm{RT}$ modeling is done at high spectral resolution as it is provided by SCIATRAN.

We have demonstrated also that employing the uncoupled atmospheric RT model to simulate satellite measurements of the reflected radiation over the oceanic sites can lead to systematic errors in the spectral range 400$550 \mathrm{~nm}$. These error are caused by the vertical inhomogeneity of the inherent optical parameters and can be significant 


\begin{tabular}{|l|l|l|l|}
\hline matter & surface conc. (0 m) & max. conc. at () m & vertical gradient per $\mathrm{m}$ \\
\hline TChla & $0.165 \mathrm{mg} / \mathrm{m}^{3}$ & $0.741 \mathrm{mg} / \mathrm{m}^{3}$ at $30 \mathrm{~m}$ & $0.0192 \mathrm{mg} / \mathrm{m}^{3}$ \\
\hline small particles & $0.072 \mathrm{mg} / \mathrm{m}^{3}$ & $0.373 \mathrm{mg} / \mathrm{m}^{3}$ at $30 \mathrm{~m}$ & $0.0100 \mathrm{mg} / \mathrm{m}^{3}$ \\
\hline large particles & $0.099 \mathrm{mg} / \mathrm{m}^{3}$ & $0.478 \mathrm{mg} / \mathrm{m}^{3}$ at $39 \mathrm{~m}$ & $0.0097 \mathrm{mg} / \mathrm{m}^{3}$ \\
\hline
\end{tabular}
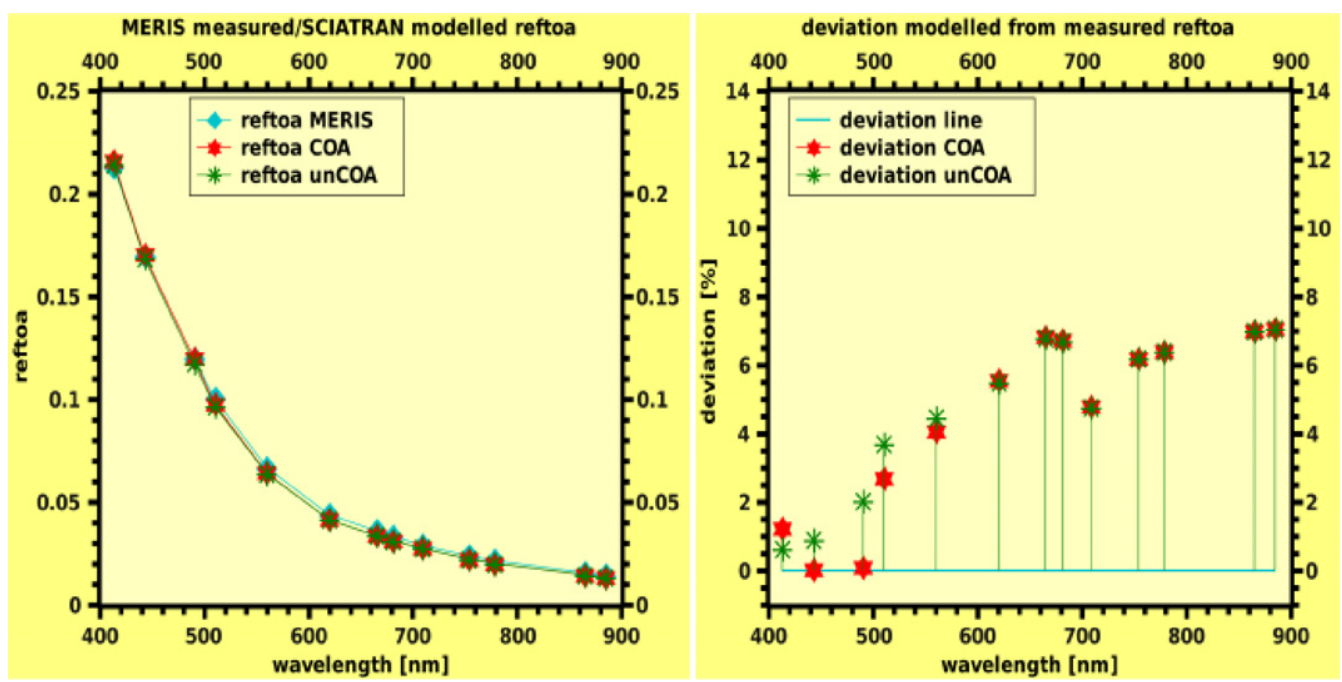

Fig. 7. The same as in Fig. 4, but for October 10, 2006. The mean deviation of COA and unCOA is $4.5 \%$ and $4.8 \%$, respectively.

if e.g. the vertical profile of the chlorophyll concentration shows strong dependence on the depth.

The SCIATRAN software package is under further development. We are working now under implementation of inelastic scattering processes such as vibrational Raman scattering and fluorescence of dissolved organic matter and chlorophyll-a. We plan that the following SCIATRAN versions will be also freely available to users. It is also planned further comparisons of the extended SCIATRAN model predictions to in-situ measurements of radiative quantities. In particular, data from the transatlantic cruise Ant XXIV/ 4 with RV Polarstern in April/May 2008 will be used. These data provide an information on the inherent optical properties as well as on the light field within and above the water.

The SCIATRAN 3.1 code has been written in FORTRAN 95. The main target computer platform is Intel/ AMD PC under LINUX operating system. At this platform, the program is configured to work with ifort, g95, and gfortran compilers, where other computer platforms/ compilers can also be used. The developed software package alone with detailed User's Guide are freely distributed at http://www.iup.physik.uni-bremen.de/sciatran. We hope that the presented software package can be of great importance predominantly for non-expert in radiative transfer users that need to apply radiative transfer calculations to own scientific work.

\section{Uncited references}

Adams and Kattawar (1993), Baker and Frouin (1987), Barichello et al. (2000), Blattern et al. (1974), Brine and Iqbal (1983), Deirmendjian (1963), Elterman (1968), Ge et al. (1993), Gordon and Brown (1973), Gordon et al. (1975), Gordon and Castano (1987), Gordon (1987), Gordon (1989), Gordon (1989), Gordon (1992), Harrison and Coombes (1988), Jin and Stamnes (1994), Jonasz and Fournier (2007), Kattawar and Adams (1989), Kattawar and Adams (1990), Kattawar and Xu (1992), Kattawar and Adams (1992), Kirk (1981), Mie (1908), Mobley and Preisendorfer (1988), Mobley (1988), Mobley (1989), Mobley (1994), Morel and Gentili (1991), Morel and Gentili (1993), Plass and Kattawar (1969), Plass and Kattawar (1972), Plass et al. (1975), Preisendorfer and Mobley (1986), Preisendorfer (1988), Stamnes and Swanson (1981), Stamnes and Dale (1981), Stavn and Weidemann (1988), Stavn and Weidemann (1992), Tanré et al. (1979) and Twardowski et al. (2007).

\section{Acknowledgements}

The authors want to thank ESA for providing MERIS level 1 and level 2 data, and AERONET team for aerosol information. We are grateful to the scientists around the 
BOUSSOLE project, especially to the director David Antoine for providing BOUSSOLE in-situ data. This work has in part been funded by HGF \& AWI (projects PHYTOOPTICS and ESSReS), German Aerospace Center (DLR), University and State of Bremen,

\section{References}

Adams, C., Kattawar, G. Effect of volume scattering function on the errors induced when polarization is neglected in radiance calculations in an atmosphere-ocean system. Appl. Opt. 20, 4610-4617, 1993.

Anikonov, A.S., Ermolaev, S.Y. On diffuse light reflection from a semiinfinite atmosphere with a highly extended phase function. Vestnik LGU 7, 132-137, 1977.

Antoine, D., d'Ortenzio, F., Hooker, S.B., Bécu, G., Gentili, B., Taillez, D., Scott, A.J. Assessment of uncertainty in the ocean reflectance determined by three satellite ocean color sensors (MERIS, SeaWiFS and MODIS-A) at an offshore site in the Mediterranean Sea (BOUSSOLE project). J. Geophys. Res. 113, C07013, doi:10.1029/ 2007JC004472, 2008.

Baker, K., Frouin, R. Relation between photosynthetically available radiation and total insolation at the ocean surface under clear skies. Limnol. Oceanogr. 32, 1370-1377, 1987.

Barichello, L.B., Garcia, R.D.M., Siewert, C.E. Particular solutions for the discrete-ordinates method. J. Quant. Spectr. Radiat. Transfer 64, 219-226, 2000.

Bezy, J.L., Delwart, S., Rast, M. MERIS - A new generation of oceancolour sensor onboard envisat. ESA Bull. 103, 48-56, 2000.

Blattern, W., Horak, H., Collins, D., Wells, M. Monte Carlo studies of the sky radiation at twilight. Appl. Opt. 13, 534, 1974.

Born, M., Wolf, E. Principles of Optics, 2nd ed Pergamon press, Oxford, London, Edinburgh, New York, Paris, Frankfurt, 1964.

Bovensmann, H., Burrows, J.P., Buchwitz, M., Frerick, J., Noël, S., Rozanov, V.V., Chance, K.V., Goede, A.P.H. SCIAMACHY: Mission objectives and measurement modes. J. Atmos. Sci. 56, 127-149, 1999.

Bracher, A., Vountas, M., Dinter, T., Burrows, J.P., Roettgers, R., Peeken, I. Quantitative observation of cyanobacteria and diatoms from space using PhytoDOAS on SCIAMACHY data. Biogeosciences 6, 751-764, 2009.

Brine, D., Iqbal, M. Diffuse and global solar spectral irradiance under cloudless skies. Sol. Energy 30, 447-453, 1983.

Buiteveld, H., Hakvoort, J.H.M., Donze, M. The optical properties of pure water. SPIE Ocean Optics XII 2258, 174-183, 1994.

Bulgarelli, B., Kisselev, V., Roberti, L. Radiative transfer in the atmosphere-ocean system: the finite-element method. Appl. Opt. 38, $1530-1542,1999$.

Chandrasekhar, S. Radiative Transfer. Oxford University Press, London, 1950.

Cox, C., Munk, W. Measurement of the roughness of the sea surface from photographs of the suns glitter. J. Opt. Soc. Am. 44 (11), 838-850, 1954.

Cox, C., Munk, W. Statistics of the sea surface derived from sun glitter. J. Marine Res. 13 (2), 198-227, 1954.

Deirmendjian, D. Scattering and polarization properties of polydisperse suspensions with partial absorption, in: Kerker, M. (Ed.), Electromagnetic Scattering. Pergamon, New York, pp. 171-189, 1963.

Elterman, L., UV, visible, and IR attenuation for altitudes to $50 \mathrm{~km}$, Report. AFCRL-68-0153, U.S. Air Force Cambridge Research Laboratory, Bedford, Mass, 1968

Evtyushenko, A.M., Kiyachenko, Y.F. Determination of the dependence of liquid refractive index on pressure and temperature. Opt. Spectrosc. 52, 56-58, 1982.

Farinato, R.S., Roswell, R.L. New values of the light scattering depolarization and anisotropy of water. J. Chem. Phys. 65, 593-595, 1976.

Fell, F., Fischer, J. Numerical simulation of the light field in the atmosphere-ocean system using the matrix-operator method. J. Quant. Spectr. Radiat. Transfer 69, 351-388, 2001.
Ge, Y., Gordon, H.R., Voss, K. Simulation of inelastic- scattering contributions to the irradiance field in the oceanic variation in Fraunhofer line depths. Appl. Opt. 32, 4028-4036, 1993.

Gordon, H.R., Brown, O. Irradiance reflectivity of a flat ocean as a function of its optical properties. Appl. Opt. 12, 1549-1551, 1973.

Gordon, H.R. Simple calculation of the diffuse reflectance of the ocean. Appl. Opt. 12 (12), 2803-2804, 1973.

Gordon, H.R., Brown, O., Jacobs, M. Computed relationships between the inherent and apparent optical properties of a flat homogeneous ocean. Appl. Opt. 14, 417-427, 1975.

Gordon, H.R., Castano, D. Coastal zone color scanner atmospheric correction algorithm: multiple scattering effects. Appl. Opt. 26, 2111, 1987.

Gordon, H.R. A bio-optical model describing the distribution of irradiance at the sea surface resulting from a point source embedded in the ocean. Appl. Opt. 26, 4133-4148, 1987.

Gordon, H.R. Can the Lambert-Beer law be applied to the diffuse attenuation coefficient of ocean water? Limnol. Oceanogr. 34, 13891409, 1989

Gordon, H.R. Dependence of the diffuse reflectance of natural waters on the sun angle. Limnol. Oceanogr. 34, 1484-1489, 1989.

Gordon, H.R. Diffuse reflectance of the ocean: influence of nonuniform phytoplankton pigment profile. Appl. Opt. 31, 2116-2129, 1992.

Gottwald, M. SCIAMACHY, Monitoring the Changing Earth's Atmosphere, DLR. Institute fuer Methodik der Fernerkundung, 2006.

Haltrin, V.I. Absorption and scattering of light in natural waters, in: Kokhanovsky, A.A. (Ed.), Light Scattering Reviews. Springer, Praxis Publishing, Chichester, UK, pp. 445-486, 2006.

Harrison, A., Coombes, C. Angular distribution of clear sky short wavelength radiance. Sol. Energy 40, 57-69, 1988.

He, Xianqiang, Bai, Yan, Zhu, Qiankun, Gong, Fang A vector radiative transfer model of coupled oceanatmosphere system using matrixoperator method for rough sea-surface. J. Quant. Spectr. Radiat. Transfer 111, 1426-1448, doi:10.1016/j.jqsrt.2010.02.014, 2010.

Jin, Z., Stamnes, K. Radiative transfer in nonuniformly refracting layered media: atmosphere-ocean system. Appl. Opt. 33 (3), 431-442, 1994.

Jin, Z., Charlock, T.P., Rutledge, K., Stamnes, K., Wang, Y. Analytical solution of radiative transfer in the coupled atmosphere ocean system with a rough surface. Appl. Opt. 45 (28), 7443-7455, 2006.

Jonasz, M., Fournier, G.R. Light Scattering by Particles in Water. Theoretical and Experimental Foundations. Academic Press, Elsevier Inc, 2007.

Kattawar, G., Adams, C. Stokes vector calculations of the submarine light field in an atmosphere-ocean with scattering according to a Rayleigh phase matrix: effect of interface refractive index on radiance and polarization. Limnol. Oceanogr. 34, 1453-1472, 1989.

Kattawar, G., Adams, C., Errors in radiance calculations induced by using scalar rather than Stokes vector theory in a realistic atmosphere-ocean system, In: Ocean Optics vol. X, (eds.) by R.W. Spinrad, Proc. Soc. Photo-Opt. Instrum. Eng. 1302, pp. 2-12, 1990.

Kattawar, G., Xu, X. Filling-in of Fraunhofer lines in the ocean by Raman scattering. Appl. Opt. 31, 1055-1065, 1992.

Kattawar, G., Adams, C. Errors induced when polarization is neglected in radiance calculations for an atmosphere-ocean system. In: Estep, L. (Ed.), Optics for the Air-Sea Interface: Theory and Measurement. Proc. Soc. Photo-Opt. Instrum. Eng. 1749, pp. 2-22, 1992.

Kirk, J. Monte Carlo procedure for simulating the penetration of light into natural waters, Div. Plant Industry Technical Paper 36, Commonwealth Scientific and Industrial Research Organization, Canberra, Australia, 1981.

Kokhanovsky, A.A., Sokoletsky, L.G. Reflection of light from semiinfinite absorbing turbid media. Part 2: Plane albedo and reflection function. Color Res. Appl. 31, 498-509, 2006.

Kokhanovsky, A.A., Budak, V.P., Cornet, C., Duan, M., Emde, C., Katsev, I.L., Klyukov D.A., Korkin, S.V., Labonnote, L.C., Min, Q., Nakajima, T., Ota, Y., Prikhach, A.P., Rozanov, V.V., Yokota, T., Zege, E.P. Benchmark results in vector atmospheric radiative transfer. J. Quant. Spectr. Radiat. Transfer 111, 1931-1946, 2010. 
Kopelevich, O.V. Small-parameter model of optical properties of seawater, in: Monin, A.S. (Ed.), Ocean Optics, Physical ocean optics, vol. 1. Nauka, Moscow (in Russian), pp. 208-234, 1983.

Lepple, F.K., Millero, F.J. The isothermal compressibility of seawater near one atmosphere. Deep-Sea Res. 18, 1233-1254, 1971.

McNeil, G.T. Metrical fundamentals of underwater lens systems. Opt. Eng. 16, 128-139, 1977.

Mie, G. Beitre zur Optik trber Medien, speziell Kolloidalen MetallLsungen. Ann. Phys. 25, 377-445, 1908.

Mobley, C.D., Preisendorfer, R. A numerical model for the computation of radiance distributions in natural waters with wind-roughened surfaces, NOAA Tech. Memo. ERL PMEL-75(NTIS PB88-192703), Pacific Marine Environmental Laboratory, Seattle, Wash., 1988.

Mobley, C.D. A numerical model for the computation of radiance distributions in natural waters with wind-roughened surfaces, part II: user's guide and code listing, NOAA Tech. Memo. ERL PMEL81(NTIS PB88-246871), Pacific Marine Environmental Laboratory, Seattle, Wash., 1988.

Mobley, C.D. A numerical model for the computation of radiance distributions in natural waters with wind-roughened surfaces. Limnol. Oceanogr. 34, 1473-1483, 1989.

Mobley, C.D., Gentili, B., Gordon, H.R., Jin, Z., Kattawar, G.W., Morel, A., Reinersman, P., Stamnes, K., Stavn, R.H. Comparison of numerical models for computing underwater light fields. Appl. Opt. 32 (36), 7484-7504, 1993.

Mobley, C.D. Light and water. Radiative transfer in natural waters. Academic Press, Inc, 1994.

Mobley, C.D., Sundman, L.K. Hydrolight - Ecolight 5.0 users' guide, Tech. Rep., Sequoia Scientific, Inc., Bellevue, WA. First Printing December 2008.

Mobley, C.D., Sundman, L.K. Hydrolight - Ecolight 5.0, Technical Documentation. Tech. Rep., Sequoia Scientific, Inc., Bellevue, WA. First Printing December 2008.

Morel, A. Optical properties of pure water and pure seawater, in: Jerlov, N.G., Nielson, E.S. (Eds.), Optical Aspects of Oceanography. Academic, New York, pp. 1-24, 1974.

Morel, A., Gentili, B. Diffuse reflectance of oceanic waters: Its dependence on Sun angle as influenced by the molecular scattering contribution. Appl. Opt. 30 (30), 4427-4438, 1991.

Morel, A., Gentili, B. Diffuse reflectance of oceanic waters. II. Bidirectional aspects. Appl. Opt. 32 (33), 6864-6879, 1993.

Morel, A., Maritorena, S. Bio-optical properties of oceanic waters: a reappraisal. J. Geophy. Res. 106 (C4), 7163-7180, 2001.

Nakajima, T., Tanaka, M. Effect of wind generated waves on the transfer of solar radiation in the atmosphere-ocean system. J. Quant. Spectr. Radiat. Transfer 29, 521-537, 1983.

O'Conner, C.L., Schlupf, J.P. Brillouin scattering in water: the LandauPaszek ratio. J. Chem. Phys. 47, 3138, 1967.

Ota, Y., Higurashi, A., Najajima, T., Yokota, T. Matrix formulations of radiative transfer including the polarization effect in a coupled atmosphere ocean system. J. Quant. Spectr. Radiat. Transfer 111, 878-894, 2010.

Petzold, T.J. Volume scattering functions for selected ocean waters, SIO Ref. 72-78, Scripps Institute of Oceanography, Visibility Laboratory, San Diego, CA, 1972.

Plass, G., Kattawar, G. Radiative transfer in an atmosphere-ocean system. Appl. Opt. 8, 455-466, 1969.

Plass, G., Kattawar, G. Monte-Carlo calculations of radiative transfer in the earth's atmosphere ocean system: I. Flux in the atmosphere and ocean. J. Phys. Oceanogr. 2, 139-145, 1972.

Plass, G., Kattawar, G., Guinn, J.Jr. Radiative transfer in the earth's atmosphere and ocean: influence of ocean waves. Appl. Opt. 14, 1924 1936, 1975.

Pope, R.M., Fry, E.S. Absorption spectrum (380-700 nm) of pure water: II. Integrating cavity measurements. Appl. Opt. 36, 8710-8723, 1997.

Preisendorfer, R., Mobley, C.D. Albedos and glitter patterns of a windroughened sea surface. J. Phys. Oceanogr. 16, 1293-1316, 1986.
Preisendorfer, R. Eigenmatrix representations of radiance distributions in layered natural waters with wind-roughened surfaces, NOAA Tech. Memo. ERL PMEL-76(NTIS PB88-188701), Pacific Marine Environmental Laboratory, Seattle, Wash., 1988.

Prieur, L., Sathyendranath, S. An optical classification of coastal and oceanic waters based on the specific absorption curves of phytoplankton pigments, dissolved organic matter, and other particulate materials. Limnol. Oceanogr. 26, 671-689, 1981.

Rozanov, A. SCIATRAN 2.X: Radiative transfer model and retrieval software package, $<$ http://www.iup.uni-bremen.de/sciatran/>, 2008.

Rozanov, V.V., Rozanov, A.V. Generalized form of the direct and adjoint radiative transfer equations. J. Quant. Spectr. Radiat. Transfer 104, 155-170, 2007.

Rozanov, A., Rozanov, V.V., Buchwitz, M., Kokhanovsky, A., Burrows, J.P. SCIATRAN 2.0 - A new radiative transfer model for geophysical applications in the $175-2400 \mathrm{~nm}$ spectral region. Adv. Space Res. 36, 1015-1019, 2005.

Rozanov, V.V., Buchwitz, M., Eichmann, K.-U., de Beek, R., Burrows, J.P. Sciatran - a new radiative transfer model for geophysical applications in the $240-2400 \mathrm{~nm}$ spectral region: the pseudo-spherical version. Adv. Space Res. 29, 1831-1835, 2002.

Schulz, F.M., Stamnes, K., Weng, F. VDISORT: an improved and generalized discrete ordinate method for polarized (vector) radiative transfer. J. Quant. Spectr. Radiat. Transfer 61 (1), 105-122, 1999.

Schulz, F.M., Stamnes, K. Angular distribution of the Stokes vector in a plane-parallel, vertically inhomogeneous medium in the vector discrete ordinate radiative transfer (VDISORT) model. J. Quant. Spectr. Radiat. Transfer 65 (4), 609-620, 2000.

Shifrin, K.S. Physical optics of ocean water AIP Translation Series. Amer. Inst. Phys., New York, pp. 285. 1988.

Siewert, C.E. On the equation of transfer relevant to the scattering of polarized light. Astrophys. J. 245, 1080-1086, 1981.

Siewert, C.E. On the phase matrix basic to the scattering of polarized light. Astron. Astrophys. 109, 195-200, 1982.

Siewert, C.E. A discrete-ordinate solution for radiative-transfer models that include polarization effects. J. Quant. Spectr. Radiat. Transfer 64, 227-254, 2000.

Stamnes, K., Swanson, R.A. A new look at the discrete ordinate method for radiative transfer calculations in anisotropically scattering atmospheres. J. Atmos. Sci. 38, 387-399, 1981.

Stamnes, K., Dale, H. A new look at the discrete ordinate method for radiative transfer calculations in anisotropically scattering atmospheres. II: Intensity computations. J. Atmos. Sci. 38, 2696-2706, 1981.

Stamnes, K., Tsay, S-Chee, Wiscombe, W., Jayaweera, K. Numerically stable algorithm for discrete-ordinate-method radiative transfer in multiple scattering and emitting layered media. Appl. Opt. 27, 25052508, 1988.

Stavn, R., Weidemann, A. Optical modeling of clear oceanlight fields: Raman scattering effects. Appl. Opt. 27, 4002-4011, 1988.

Stavn, R., Weidemann, A. Raman scattering in ocean optics: quantitative assessment of internal radiant emission. Appl. Opt. 31, 1294-1303, 1992.

Tanré, D., Herman, M., Deschamps, R., deLeffe, A. Atmospheric modeling for space measurements of ground reflectances including bi-directional properties. Appl. Opt. 18, 3587-3594, 1979.

Thomas, G.E., Stamnes, K. Radiative Transfer in the Atmosphere and Ocean. Cambridge University Press, 1999.

Twardowski, M.S., Claustre, H., Freeman, S.A., Stramski, D., Huot, Y. Optical backscattering properties of the "clearest" natural waters. Biogeosciences 4, 1041-1058, 2007.

Vountas, M., Dinter, T., Bracher, A., Burrows, J.P., Sierk, B. Spectral studies of ocean water with space-borne sensor SCIAMACHY using differential optical absorption spectroscopy (DOAS). Ocean Sci. 3, 429-440, 2007.

Zhai, P.W., Hu, Y., Chowdhary, J., Trepte, C.R., Lucker, P.L., Josset, D.B. A vector radiative transfer model for coupled atmosphere and ocean systems with a rough interface. J. Quant. Spectrosc. Radiat. Transfer 111, 1025-1040, 2010.
1026

1027

1028

1029

1030

1031

1032

1033

1034

1035

1036

1037

1038

1039

1040

1041

1042

1043

1044

1045

1046

1047

1048

1049

1050

1051

1052

1053

1054

1055

1056

1057

1058

1059

1060

1061

1062

1063

1064

1065

1066

1067

1068

1069

1070

1071

1072

1073

1074

1075

1076

1077

1078

1079

1080

1081

1082

1083

1084

1085

1086

1087

1088

1089

1090

1091

1092

1093 